\title{
Antibacterial activity and hormetic response of silver nanoparticles synthesized using leaflet extract of wheat (Triticum aestivum) and rice (Oryza sativa) crop plants
}

\author{
Vikas Pahal ${ }^{1 *}$ (D), Pankaj Kumar², Parveen $\mathrm{Kumar}^{3}$ (D), Vinod Kumar ${ }^{4}$ \\ ${ }^{1}$ Department Of Microbiology, Dolphin PG College of Science and Agriculture, Chunni Kalan, Fatehgarh Sahib, Punjab, India. \\ ${ }^{2}$ Department Of Microbiology, Dolphin PG Institute of Biomedical and Natural Sciences, Dehradun, India. \\ ${ }^{3}$ Bio-nanotechnology Lab, Central Scientific Instruments Organization, Chandigarh, India. \\ ${ }^{4}$ Department of Pharmacology and toxicology, National Institute of Pharmaceutical Education and Research, S.A.S. Nagar, Mohali, India.
}

\section{ARTICLE INFO \\ Article history: \\ Received on: August 24, 2021 \\ Accepted on: October 26, 2021 \\ Available Online: February 15, 2022}

Key words:

AgNPs, antibacterial effect, crop plants, hormetic response, XTT colorimetric assay

\begin{abstract}
The application of silver nanoparticles (AgNPs)-based antibacterial therapeutics has emerged as a feasible alternative to traditional antibiotic therapy due to cost-effectiveness and lower possibility of non-evolution of resistant strains. In the present paper, the aqueous extract of wheat (Triticum aestivum) and rice (Oryza sativa) leaflets were used for the fabrication of well-dispersed AgNPs of average size 19.11 and $33.85 \mathrm{~nm}$, respectively, under the controlled condition of $\mathrm{pH} 10.0 \pm 0.1$ and temperature $80^{\circ} \mathrm{C} \pm 1{ }^{\circ} \mathrm{C}$. This bottom-up approach of AgNPs production was simple, eco-friendly, inexpensive, and highly reproducible. The synthesized AgNPs were characterized by UV-Visible spectroscopy, dynamic light scattering, fourier transform infrared spectroscopy, high-resolution transmission electron microscopy, and energy-dispersive X-ray. Agarwell diffusion and (2, 3-bis [2-methyloxy-4-nitro-5-sulfophenyl]-2H-tetrazolium-5-carboxanilide) (XTT) colorimetric methods revealed the bactericidal potential of synthesized AgNPs against Gram-positive and Gram-negative pathogenic bacteria with minimum inhibitory concentration (MIC) values ranges from 125 to $500 \mu \mathrm{g} / \mathrm{ml}$. AgNPs presented better potency against Gram-negative bacteria compared to Gram-positive bacteria. Interestingly, Staphylococcus aureus, Klebsiella pneumoniae, and Escherichia coli demonstrated a hormetic response (positive stimulated growth) at a sub-lethal concentration $(<7.81 \mu \mathrm{g} / \mathrm{ml})$ of AgNPs, which were $0.39 \%-1.56 \%$ of MIC values of the respective bacterial strains.
\end{abstract}

\section{INTRODUCTION}

One of the striking and developing areas of nanotechnology deals with the production of metallic nanoparticles (NPs) of variable shapes, sizes, and chemical compositions. Owing to their ultrasmall size, elevated reactivity, and substantially large surface area-to-volume ratio, NPs display astonishing physicochemical, optical, magnetic, thermal, electronic, and biological properties markedly different from their respective bulk counterparts [1-3]. These inherent properties have tremendous applications

\footnotetext{
*Corresponding Author

Vikas Pahal, Department Of Microbiology, Dolphin PG College of Science and Agriculture, Chunni Kalan, Fatehgarh Sahib, Punjab, India.

E-mail:vikaspahal3@gmail.com
}

in various fields of science and technology such as electronics, biotechnology, sensors, drug delivery, DNA labeling, cosmetics, coatings, packaging, etc. [4,5]. Among various metallic NPs, nanocrystalline silver or silver nanoparticles (AgNPs) have found incredible applications in the field of catalysis [2], detection, bioremediation $[1,3]$, diagnostic, biosensors, micro- and optoelectronics [4], antimicrobials, medical implants, and therapeutics $[6,7]$; and hence account for more than $55 \%$ of total nano-materialbased commercial products [8].

Various physical, chemical, and hybrid methods are developed for the fabrication of AgNPs. However, the key challenges that remain with these methods are their toxicity, expensiveness, high energy consumption, and time along with several procedural complications [9]. Green chemistry procedures, which involve the synthesis of NPs using biological entities like bacteria, (c) 2022 Pahal et al. This is an open access article distributed under the terms of the Creative Commons Attribution License -NonCommercial-ShareAlike Unported License (http://creativecommons.org/licenses/by-nc-sa/3.0/). 
cyanobacteria, algae, fungi, and plants, represent alternatives to these harmful procedures [10-12]. Furthermore, employing plants for the production of AgNPs can be a more advantageous and economical alternative over other biological processes that involve microorganisms. First, it lowers down the cost of isolation and maintenance of microorganisms in the culture media $[13,14]$. Second, plants have a wide variety of metabolites with tremendous reduction potential and capping capacity $[10,15]$. Third, they are easily available, much safer to handle, more biocompatible, and environmentally benign $[10,16]$.

Pathogenic bacteria and their resistance strains are responsible for the rise in mortality and morbidity cases worldwide. This has triggered the need to develop new and improved therapeutic agents, which is presently the most urgent priority in biomedical research [17]. The use of AgNPs-based antibacterial therapeutics has emerged as a feasible alternative to traditional antibiotic therapy due to cost-effectiveness, broad-spectrum antimicrobial effect, non-toxicity toward human cells (concentration-based), and the lower possibility for the development of resistance because of their non-receptor mediated inhibitory mechanism [18,19]. Owing to the various advantages of plant-based fabrication of AgNPs, several plants have been successfully used for efficient and rapid extracellular synthesis of AgNPs. However, the problem remains with the production of small-sized NPs of controlled shapes and sizes. It has been well documented that smaller NPs have a greater potential to kill microbes than large-sized NPs [8].

In this paper, we report a clean production of AgNPs with controlled morphology using aqueous extract of leaflets of Triticum aestivum (wheat) and Oryza sativa (rice) plants, which are cereal grass of family Gramineae and are major crops of Asia. Aqueous extract of T. aestivum and $O$. sativa leaflets have found to possess phenolics compounds, lignins, tannins, flavonoids, alkaloids, carotenoids, vitamins, terpenoids, amino acids, carbohydrates, and antioxidant activity [20,21]. Furthermore, the extract of wheatgrass (2-3-week leaflets) was also reported to possess anti-inflammatory, antidiabetic, antibacterial, and immunomodulatory activities [21-23]. Different characterization techniques, such as FTIR, dynamic light scattering (DLS), UV-Vis spectroscopy, and high-resolution transmission electron microscopy (HRTEM), were utilized to evaluate the reaction results. The synthesized AgNPs were further investigated to assess their antibacterial efficiency against ten experimental bacterial strains using agar-well diffusion and XTT colorimetric assays.

\section{MATERIAL AND METHODS}

\subsection{Chemicals}

Analytical grade growth media and chemicals were used in the present investigation. HPLC-grade water, Mueller-Hinton agar (MHA), and broth (MHB) were obtained from Hi-Media Pvt. Ltd, Chandigarh (U.T), India. Silver nitrate $\left(\mathrm{AgNO}_{3}\right)$, XTT salt, and menadione (purity $>99.0 \%$ ) were procured from Sigma-Aldrich, India.

\subsection{Biosynthesis of AgNPs}

Wheat (variety 2,967) and rice (variety 1,121) were grown in our agriculture laboratory. Approximately 10 gm leaflets of 2-3 weeks long wheat (T. aestivum) and rice (O. sativa) plants were cut into fine pieces and added to $50 \mathrm{ml}$ of HPLC purified water in two separate $100 \mathrm{ml}$ conical flasks. The solutions were boiled for 5-7 minutes and then cooled at room temperature. After cooling, both extracts were filtered through filter paper (Whatman No. 1) and then centrifuged for 6-8 minutes at 10,000 rpm to remove the plant debris. This impurities-free supernatant was used for the synthesis of AgNPs. For biosynthesis of AgNPs, $10 \mathrm{ml}$ of wheat and rice leaflet extracts was added to $90 \mathrm{ml}$ of $3 \mathrm{mM} \mathrm{AgNO}_{3}$ solution in two separate $250 \mathrm{ml}$ conical flasks with continuous stirring on a magnetic stirrer at constant temperature $\left(80.0^{\circ} \mathrm{C} \pm 1{ }^{\circ} \mathrm{C}\right)$ and $\mathrm{pH}$ $(10.0 \pm 0.1)$. The color of both the solutions changed to reddishbrown within 2-3 minutes, which indicated the completion of the reaction mechanism for the formation of AgNPs. Both the solutions were further kept for 2-4 hours of incubation period at room temperature, and after that centrifuged for 15-20 minutes at $16,000 \mathrm{rpm}$. AgNPs were settled down and the supernatant was aseptically removed. This procedure was repeated thrice with High performance liquid chromatography (HPLC) purified water to get AgNPs without any impurity. Finally, AgNPs were resuspended in $5 \mathrm{ml}$ HPLC purified water and dispersed on a Petri-plate and dried in a vacuum oven $\left(60^{\circ} \mathrm{C}\right)$ to get AgNPs in dry form. Wheat- and rice-mediated biosynthesized AgNPs were labeled as Wheat-leaflet mediated synthesized silver nanoparticles (WNPs) and Rice-leaflet mediated synthesized silver nanoparticles (RNPs), respectively. The dried AgNPs were physically characterized and analyzed for their bactericidal properties.

\subsection{Compositional and Morphological Characterization of AgNPs}

\subsubsection{UV-visible and FTIR spectroscopy}

The ultraviolet visible absorption spectrum of synthesized AgNPs was obtained in the wavelength range of 280-660 nm using a UVvisible spectrophotometer (Thermo Fisher Scientific, India). FTIR emission spectrum of AgNPs (WNPs and RNPs) was obtained in the wavelength range of 4,000-250 $\mathrm{cm}^{-1}$ using an LF-45 fluorescence FTIR spectrophotometer (Perkin Elmer).

\subsubsection{Dynamic light scattering (DLS)}

Size distributions of synthesized AgNPs were measured with DLS equipment model Malvern Zetasizer Nanosystem (Worcestershire, U.K). Before analyzing the size distribution profile of AgNPs, the aqueous suspension of AgNPs was prepared and percolated out of a syringe-based filter unit $(0.22 \mu \mathrm{m}$ diameter $)$.

\subsubsection{HRTEM, energy-dispersive $X$-ray (EDX) spectroscopy, and SAED pattern analysis}

To analyze the structural morphology of AgNPs, HRTEM images of AgNPs were obtained and analyzed using an FEI TECNAI (G2 F20) system (200 keV) with $6 \times 10^{6}$ magnification and at a resolution of $0.2 \AA$. EDX microanalysis of synthesized AgNPs was carried out in EDX spectroscopy in conjunction with HRTEM to analyze the elemental composition of AgNPs. Selected area electron diffraction (SAED) pattern was investigated to confirm the crystalline character of synthesized AgNPs. 


\subsection{Assessment of the Bactericidal Efficiency of AgNPs}

To investigate the bactericidal efficiency of green synthesized AgNPs, five Gram-positive bacterial strains, namely Streptococcus pneumoniae [Microbial Type Culture Collection (MTCC) 1935], Micrococcus luteus (MTCC 106), Staphylococcus epidermidis (MTCC 9040), Streptococcus mutans (MTCC 890), and Staphylococcus aureus (MTCC 3160) and five Gramnegative bacterial strains, i.e., Salmonella typhimurium (MTCC 3224), Klebsiella pneumoniae (MTCC 432), Escherichia coli (MTCC 443), Enterobacter aerogenes (MTCC 2824), and Vibrio cholerae (MTCC 3904) were procured from Institute of Microbial Technology, Sector 39, Chandigarh (U.T), India.

\subsubsection{Agar-well diffusion assay}

Initially, all the bacterial strains were grown in MHB for 1620 hours at $37^{\circ} \mathrm{C} .100 \mu \mathrm{l}$ of each bacterial culture were then inoculated into $50 \mathrm{ml}$ of fresh broth for 2.5-3.5 additional hours to get mid-logarithmic phase bacteria [24]. Bacterial cultures were then centrifuged for $3,000-4,000 \mathrm{rpm}$ at $4^{\circ} \mathrm{C}$ for 10 minutes. The supernatant was removed aseptically, and the pellets were cleansed with the help of $10 \mathrm{mM}$ sodium phosphate buffer $(\mathrm{pH}=7.4)$. This procedure was repeated thrice, and finally, the bacterial pellets were resuspended in the same cold buffer. The concentration of bacteria was standardized with the optical density of bacterial aliquot at $620 \mathrm{~nm}[24,25]$, where OD $=0.20$ is equivalent to approximately $5 \times 10^{7} \mathrm{CFU} / \mathrm{ml}$. MHA plates of all the bacterial strains were prepared by spreading 100 $\mu l$ of the inoculums of each test bacteria on their respective MHA plates [25]. Five wells per plate were made with the help of a sterile borer. HPLC purified water was used to make different concentrations of AgNPs. For the assessment of the bactericidal effect of different concentrations $(2,1,0.5$, and $0.25 \mathrm{mg} / \mathrm{ml}$, respectively) of AgNPs, $90 \mu \mathrm{l}$ of AgNPs solution was injected aseptically into the agar wells, and the agar plates were then incubated for $18-24$ hours at $37^{\circ} \mathrm{C}$ for the observation of zone of inhibition (ZOI), if any. In all experiments, the antibiotic ciprofloxacin $(0.5 \mathrm{mg} / \mathrm{ml})$ was used as a positive control. All experiments were carried out in triplicate.

\subsubsection{XTT colorimetric method}

Bactericidal activity of synthesized AgNPs was also evaluated through the XTT colorimetric method [25-27]. This method is based on the principle that viable bacteria's dehydrogenase enzyme reduces sodium salt of XTT (2, 3-bis [2-methyloxy-4-nitro-5sulfophenyl]-2H-tetrazolium-5-carboxanilide) into a water-soluble formazan, whose absorbance can be measured at $490 \mathrm{~nm}$. This method evaluates the bactericidal efficacy of AgNPs by estimating the relative reduction in the production of colored formazan by the viable bacteria in the reaction media. In this experiment, $170 \mu \mathrm{l}$ of the adjusted broth cultures of different bacteria $\left(5 \times 10^{7} \mathrm{CFU} / \mathrm{ml}\right)$ were seeded into the 96-well flat-bottom microtiter plate, followed by the addition of $30 \mu \mathrm{l}$ of AgNPs (of different concentrations)
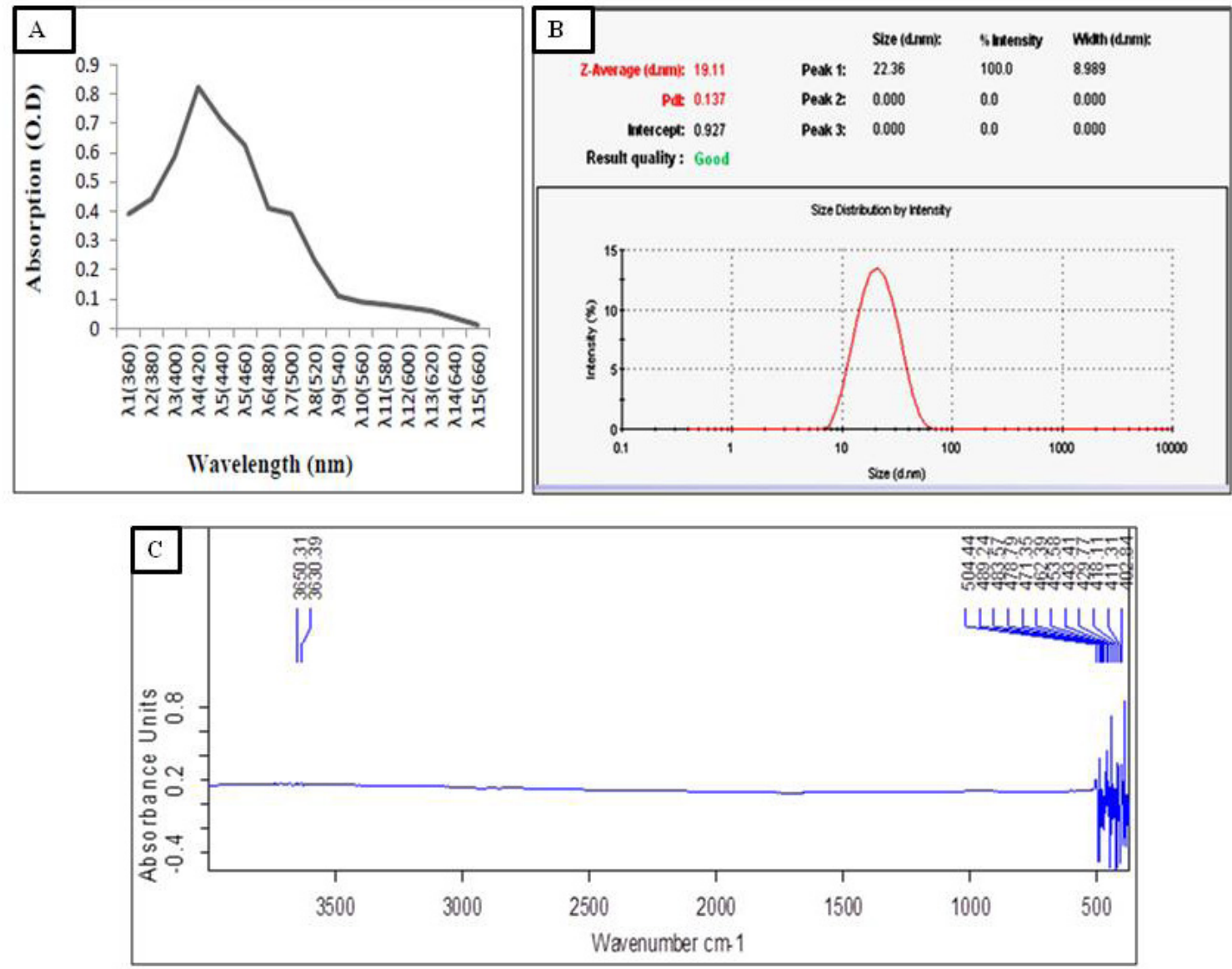

Figure 1: UV-Vis spectroscopy (A), DLS (B), and FTIR spectra (C) results of AgNPs (WNPs) synthesized from wheatgrass (T. aestivum). 

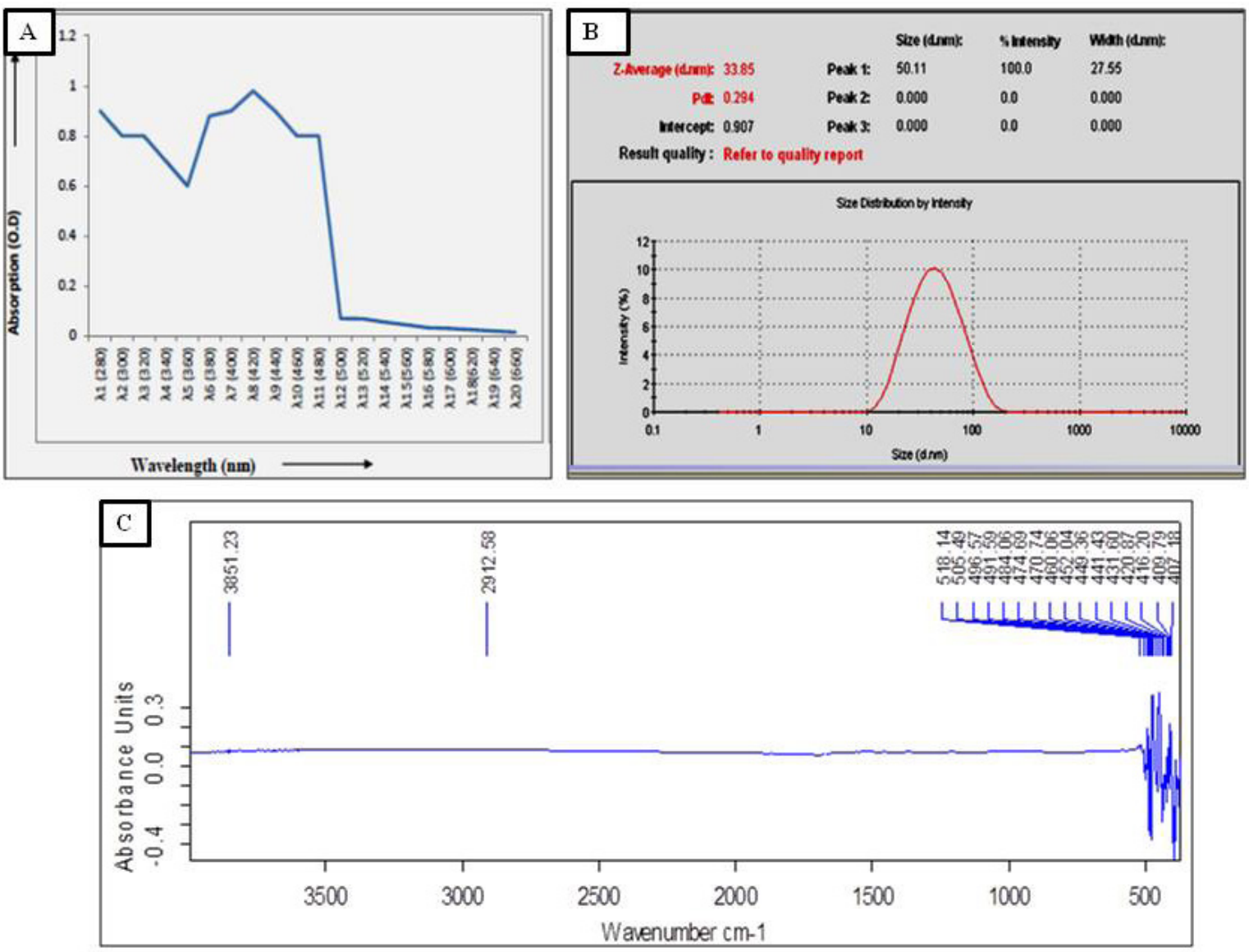

Figure 2: UV-Vis spectroscopy (A), DLS (B), and FTIR spectra (C) report of AgNPs (RNPs) synthesized from O. sativa.

solution and incubated for 15 hours at $37^{\circ} \mathrm{C}$. After completion of first incubation, $100 \mu \mathrm{l}$ of reaction material of well was shifted into a new flat-bottom microtiter plate. Simultaneously, $25 \mu 1$ of a fresh solution of XTT $(1 \mathrm{mg} / \mathrm{ml})$ and menadiaone $(1 \mathrm{mM})$ in the volume ratio of 12.5:1 was supplemented to each well with gentle mixing, and the plates were incubated at $37^{\circ} \mathrm{C}$ for $1-1.5$ hours. Finally, the absorbance of colored formazan was measured at $490 \mathrm{~nm}$. Antibiotic ciprofloxacin $(0.5 \mathrm{mg} / \mathrm{ml})$ acted as a positive control, whereas negative control was the MHB containing the respective bacterial strains in the absence of any bactericidal agent. Final readings were corrected by subtracting the absorbance of various positive blanks. Bactericidal efficiency was evaluated as the \% Rd (percentage reduction) of formazen by applying the following formula:

$\begin{aligned} & \text { Percentage } \\ & \text { reduction }(\% \mathrm{Rd})\end{aligned}=100 \%-100$
2.4.3. Absorbance in negative control

All experiments were repeated in triplicate and the final readings were calculated as a mean of percentage reduction $( \pm \mathrm{SD})$. During the evaluation of the bactericidal efficiency of AgNPs, a percentage reduction of less than $5 \%$ was considered as concentrations having no effect (NE) on bacterial growth.

\subsection{Determination of Minimum Inhibitory Concentration (MIC) and Minimum Bactericidal Concentration (MBC)}

MIC is defined as the minimum concentration of AgNPs that did not allow any bacterial growth, as conveyed by the OD results of XTT colorimetric method. However, MBC is defined as the minimal concentration of AgNPs that will not permit the growth of bacteria after sub-culturing it on MHA plates that are free from any antibiotic or bactericidal agent $[28,29]$. It was determined by sub-culturing the bacteria from those wells, which did not exhibit any appearance of bacterial growth as observed while determining the MIC values [25,30].

\section{RESULT AND DISCUSSION}

\subsection{UV-Visible and FTIR Spectroscopy Profile of AgNPs}

Absorption peak maxima were found to be near $420 \mathrm{~nm}$ (Figures 1a and 2a) in the case of both WNPs and RNPs. Wheat and rice are nutritious plants and contain various phytochemicals like flavonoids, phenols, alkaloids, proteins, etc. These phytochemicals have a strong reducing potential to reduce $\mathrm{AgNO}_{3}$ solution (colorless) to AgNPs (reddish brown) within 2-3 minutes of reaction and their stabilization in the solution. The surface plasmon excitation, which caused a change of solution color and absorption maxima near $420 \mathrm{~nm}$, is primary evidence of AgNPs formation in solution and inherent to biologically synthesized AgNPs $[3,31,32]$. 
FTIR analysis is an authoritative analyzing tool that identifies diverse functional groups and chemical bonds inherent to a molecule by generating an infrared absorption spectrum [14,33]. The obtained FTIR absorption spectra of AgNPs, as shown in Figures $1(\mathrm{C})$ and $2(\mathrm{C})$, chiefly exhibits strong reaction traces of the agrarian molecular extracts and that of silver precursor $\left(\mathrm{AgNO}_{3}\right)$. In such studies, prominent spectral bands assuring reaction success were usually exposed between wavenumber $300-650 \mathrm{~cm}^{-1}$. In the plant-mediated synthesis of AgNPs, observation of bands in the above range was mainly ascribed to stretching vibrations of various glucose rings and identified residual biomolecular traces. The obtained FTIR spectra of AgNPs clearly expose band growth in the above range of wavenumber that further intensifies in the gamut of absorption regime between $500-400 \mathrm{~cm}^{-1}$, indicating the success of the reaction and formation of AgNPs [12,29,34]. The appearance of two maxima near 430 and $415 \mathrm{~cm}^{-1}$ in the absorption spectra also indicates particles with reduced size. In such studies, maximum absorption observed near $430 \mathrm{~cm}^{-1}$ wavenumber indicates a high conversion of AgNPs from their precursor. Nevertheless, the presence of multiple bands, especially between 400 and $450 \mathrm{~cm}^{-1}$ in the spectra, suggests that particle size was significantly varying because of anisotropy existing in the reaction outcome [34]. In WNPs, double bands at 3,630 and 3,650 $\mathrm{cm}^{-1}$ were ascribed to $\mathrm{O}-\mathrm{H}$ stretching of alcohols. However, bands at 2,912 and $3,851 \mathrm{~cm}^{-1}$ in RNPs were due to the aliphatic C-H stretching of alkanes $\left(\mathrm{CH}_{2}\right)$ and presence of moisture in NPs, respectively [12,29,35]. It has been postulating that water-soluble flavonoids and proteins existing in plants are accountable for $\mathrm{Ag}^{+}-\mathrm{Ag}^{0}$ reduction and their stabilization in the solution $[12,31,32,35,36]$.

\subsection{HRTEM/DLS/EDX RESULTS}

HRTEM, SAED pattern, and EDX studies were carried out to assess the size and shape, crystallinity, and elemental analysis of synthesized AgNPs, respectively [12,31,32]. HRTEM images revealed that most of the synthesized AgNPs (WNPs and RNPs) were spherical and oval-shaped, as shown in Figures 3 and 4. In WNPs, the average size of NPs was found to be in the range of 5-40 nm (Figure 3a, b and c), whereas in the case of RNPs, the average size was observed in the range of 10-70 nm (Figure 4a, $\mathrm{b}$ and $\mathrm{c}$ ). Structural and elemental analyses of both AgNPs were further investigated using SAED and EDX spectroscopy, as shown in Figures 3 and 4. In both synthesized AgNPs, EDX spectra showed that silver was the only ingredient of NPs, as shown in Figures 3 (f) and 4 (f). Furthermore, as shown in Figures 3 (d) and 4 (d), the SAED pattern confirmed the high crystalline nature of synthesized AgNPs. The lattice profiles of WNPs and RNPs are shown in Figures 3 (e) and 4 (e), which suggests the average "d-spacing" of lattice fringes of respective interplane distance. DLS analysis of AgNPs further provided significant information about the size distribution profile of synthesized NPs (Figures $1 b$ \& $2 b$ ). The technique revealed that the average particle size of WNPs and RNPs were 19.11 and 33.85 $\mathrm{nm}$ with an approximate size distribution between 8-70 and 10-120 nm, respectively. HRTEM and DLS results corroborated our FTIR findings by exhibiting the anisotropy of reactions in both WNPs and RNPs. The size, shape, morphology, and stability of NPs depend on various parameters, including the $\mathrm{pH}$, reaction temperature, capping agents, concentration of metal precursor salt, reduction potential of a reductant, etc. Parameters such as high $\mathrm{pH}$, high temperature, and



Figure 3: HRTEM analysis results of WNPs. HRTEM image with scale bar $=100 \mathrm{~nm}(\mathrm{~A}), 50 \mathrm{~nm}(\mathrm{~B}), 2 \mathrm{~nm}(\mathrm{C})$, SAED pattern (D), line profile of lattice fringes (E), and EDX spectra $(\mathrm{F})$.

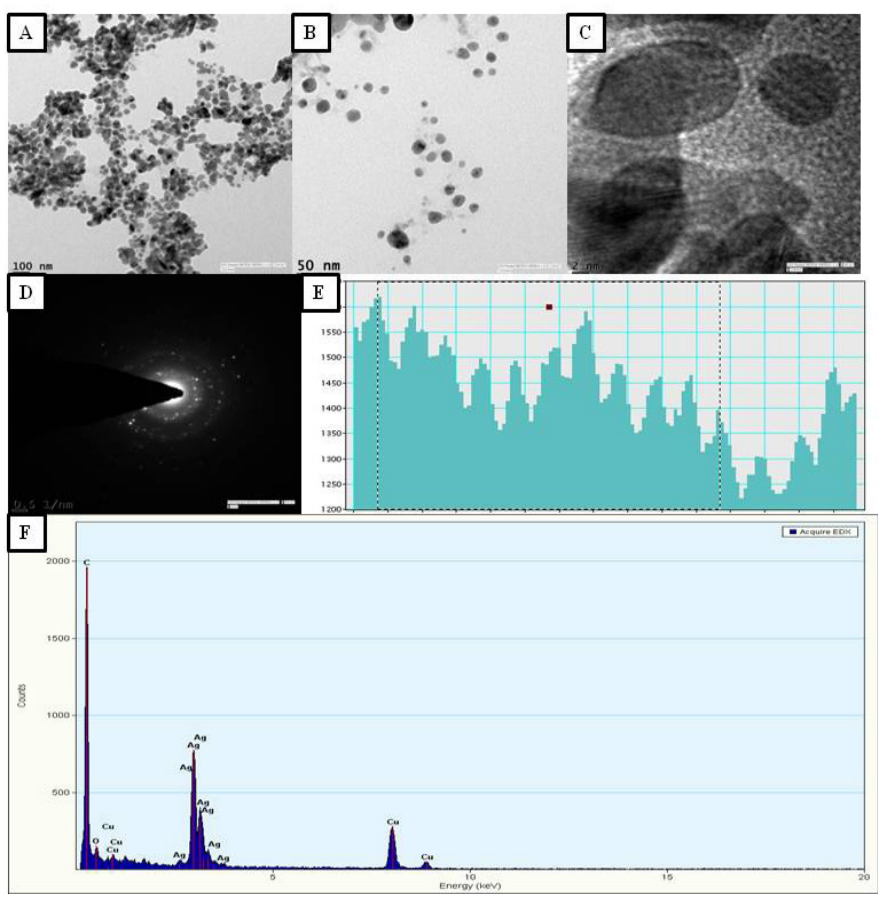

Figure 4: HRTEM analysis results of RNPs. HRTEM image with scale bar $=100 \mathrm{~nm}(\mathrm{~A}), 50 \mathrm{~nm}(\mathrm{~B}), 2 \mathrm{~nm}(\mathrm{C})$, SAED pattern (D), line profile of lattice fringes (E), and EDS spectra (F).

a lower concentration of precursor salt were found to help in the formation of small-sized NPs [37,38]. Bark, seed, fruit, seeds, root, flower, and leaves of various plants were used to synthesize AgNPs of size $0.5-250 \mathrm{~nm}$ of various shapes like the spherical, octahedron, 


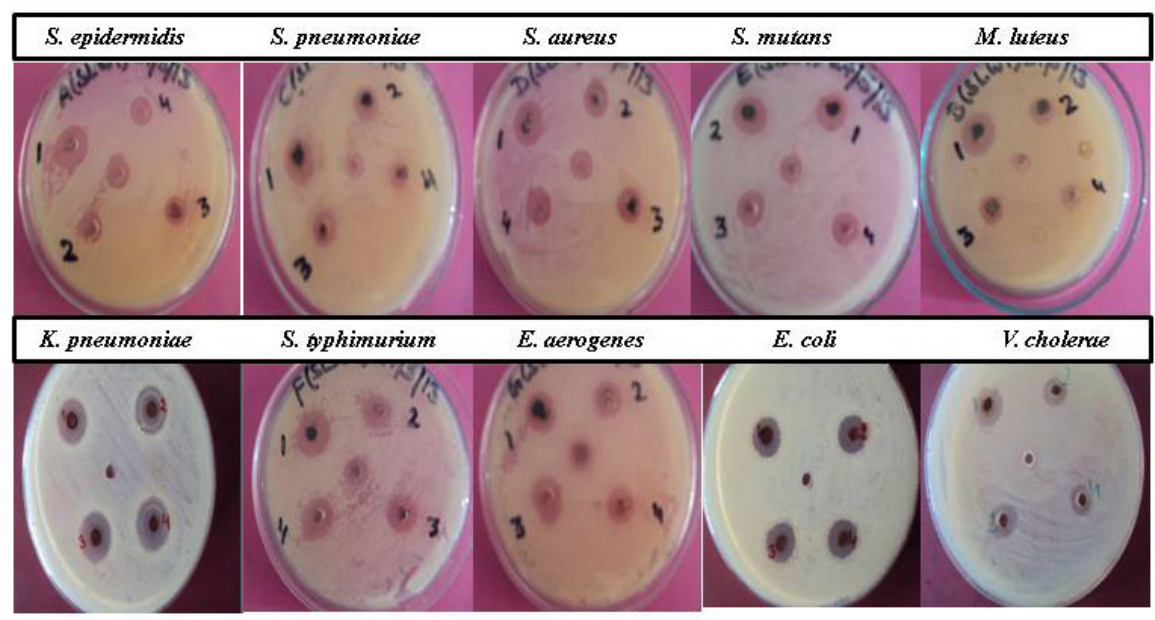

Figure 5: Agar-well diffusion assay results displaying bactericidal effect (ZOI) of WNPs against Gram-positive and Gram-negative bacteria. Different concentrations were 2.0 (1), 1.0 (2), 0.5 (3), and $0.25 \mathrm{mg} / \mathrm{ml}(4)$.

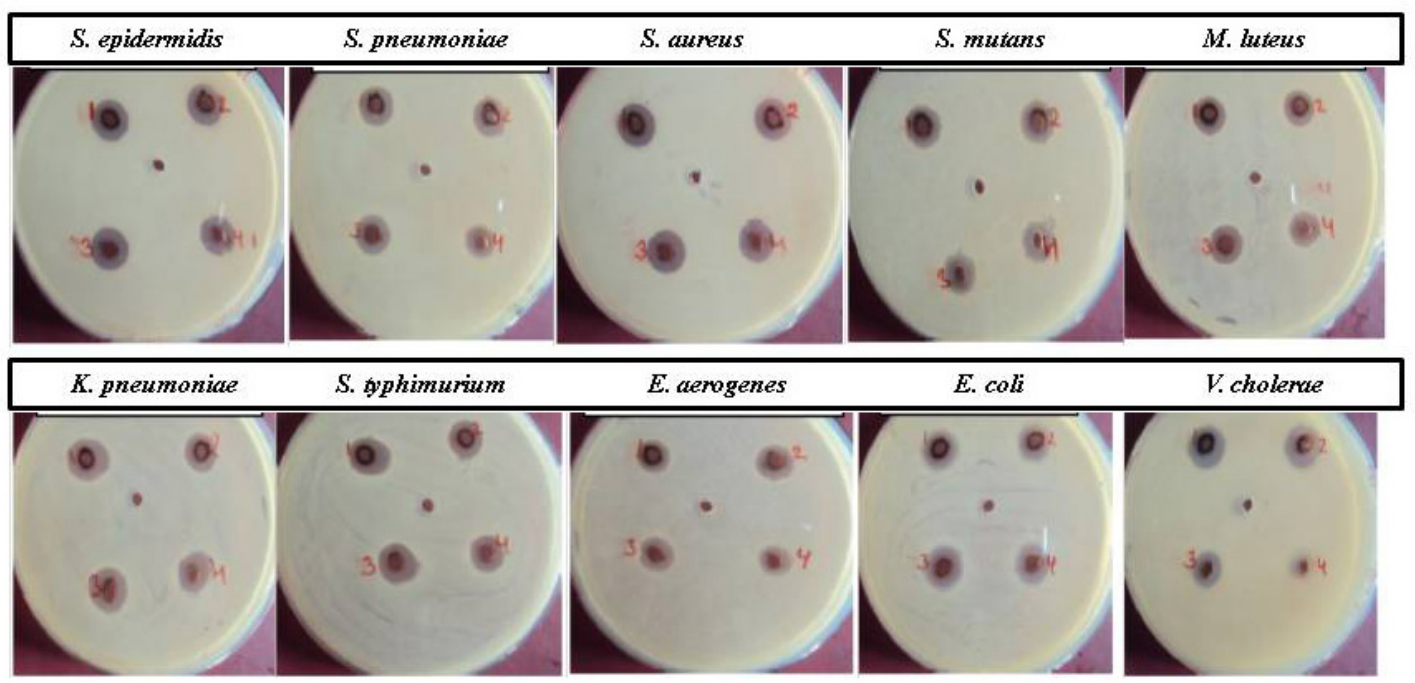

Figure 6: Agar-well diffusion assay results displaying bactericidal effect (ZOI) of RNPs against Gram-positive and Gramnegative bacteria. Different concentrations were 2.0 (1), 1.0 (2), 0.5 (3), and $0.25 \mathrm{mg} / \mathrm{ml}$ (4).

triangular, oval, rod, and face-centered cubic [11,14,15,37,38]. For example, Tribulus terrestris L., Cocos nucifera, Azadirachta indica, and Mangifera indica extract mediated synthesis of AgNPs were found to be spherical in shape, having a diameter in the range of 16-28 [15], 10-35 [39], 22 [37], and 200-350 nm [14], respectively. In the present study, AgNPs were mostly spherical, with some percentage of oval-shaped NPs and these shapes are best suited for practical applications [8].

\subsection{Assessment of the Bactericidal Effect of WNPs and RNPs}

Agar-well diffusion assay results showed that both WNPs and RNPs possessed profound antibacterial activity against all pathogenic experimental bacterial strains (Figures 5 and 6). As shown in Table 1, at a concentration of $2.0 \mathrm{mg} / \mathrm{ml}$, WNPs were most effective against Gram-positive $S$. aureus (ZOI: $7.66 \pm 0.28 \mathrm{~mm}$ ), followed by $S$. mutans (ZOI: $7.16 \pm 0.28 \mathrm{~mm}$ ), S. epidermidis (ZOI: $6.83 \pm$
$0.28 \mathrm{~mm}$ ), M. luteus (ZOI: $5.83 \pm 0.28 \mathrm{~mm}$ ), and $S$. pneumoniae (ZOI: $4.16 \pm 0.28 \mathrm{~mm}$ ). RNPs at the same concentration showed maximum antibacterial activity against $S$. aureus (ZOI: $6.83 \pm$ $0.28 \mathrm{~mm}$ ), followed by $S$. epidermidis (ZOI: $6.33 \pm 0.57 \mathrm{~mm}), \bar{S}$. pneumoniae (ZOI: $5.33 \pm 0.57 \mathrm{~mm}$ ), S. mutans (ZOI: $5.16 \pm 0.28$ $\mathrm{mm}$ ), and least effective against $M$. luteus (ZOI: $4.33 \pm 0.57 \mathrm{~mm}$ ). In the case of Gram-negative bacteria (Table 2), at $2.0 \mathrm{mg} / \mathrm{ml}$ concentration, WNPs demonstrated significant bactericidal activity against $K$. pneumoniae (ZOI: $8.83 \pm 0.28 \mathrm{~mm}$ ), followed by $S$. typhimurium (ZOI: $8.16 \pm 0.28 \mathrm{~mm})$, E. coli (ZOI: $5.83 \pm$ $0.28 \mathrm{~mm}$ ), $V$. cholerae (ZOI: $5.16 \pm 0.28 \mathrm{~mm}$ ), and least against E. aerogenes (ZOI: $5.00 \pm 0.00 \mathrm{~mm}$ ). However, RNPs exhibited maximum bactericidal effect against $K$. pneumoniae (ZOI: $6.83 \pm$ $0.28 \mathrm{~mm}$ ) and minimum against $V$. cholerae (ZOI: $4.33 \pm 0.57 \mathrm{~mm}$ ) at a concentration of $2.0 \mathrm{mg} / \mathrm{ml}$. At lower concentrations, the antibacterial effect (ZOI) of WNPs and RNPs followed a similar or slightly different trend of effectiveness. As per agar-well 
Table 1: ZOI (mean \pm SD) observed against various gram-positive bacteria by different concentrations of AgNPs (WNPs \& RNPs).

\begin{tabular}{|c|c|c|c|c|c|}
\hline \multirow{2}{*}{\multicolumn{2}{|c|}{ Bacteria }} & \multicolumn{4}{|c|}{ ZOI (mm) } \\
\hline & & $2.0 \mathrm{mg} / \mathrm{ml}$ & $1.0 \mathrm{mg} / \mathrm{ml}$ & $0.5 \mathrm{mg} / \mathrm{ml}$ & $0.25 \mathrm{mg} / \mathrm{ml}$ \\
\hline \multirow{2}{*}{$\begin{array}{l}\text { Staphylococcus } \\
\text { epidermidis }\end{array}$} & WNPs & $6.83 \pm 0.28$ & $5.33 \pm 0.57$ & $4.00 \pm 0.00$ & $2.83 \pm 0.28$ \\
\hline & RNPs & $6.33 \pm 0.57$ & $5.16 \pm 0.28$ & $3.33 \pm 0.28$ & $2.00 \pm 0.00$ \\
\hline \multirow{2}{*}{$\begin{array}{l}\text { Streptococcus } \\
\text { pneumoniae }\end{array}$} & WNPs & $4.16 \pm 0.28$ & $3.33 \pm 0.57$ & $2.00 \pm 0.00$ & $1.00 \pm 0.00$ \\
\hline & RNPs & $5.33 \pm 0.57$ & $2.83 \pm 0.28$ & $2.00 \pm 0.00$ & $1.00 \pm 0.00$ \\
\hline \multirow{2}{*}{ Staphylococcus aureus } & WNPs & $7.66 \pm 0.28$ & $5.16 \pm 0.28$ & $4.83 \pm 0.28$ & $3.16 \pm 0.28$ \\
\hline & RNPs & $6.83 \pm 0.28$ & $4.33 \pm 0.28$ & $3.0 \pm 0.00$ & $2.83 \pm 0.28$ \\
\hline \multirow{2}{*}{ Streptococcus mutans } & WNPs & $7.16 \pm 0.28$ & $5.66 \pm 0.28$ & $3.83 \pm 0.28$ & $2.33 \pm 0.57$ \\
\hline & RNPs & $5.16 \pm 0.28$ & $4.00 \pm 0.00$ & $3.16 \pm 0.28$ & $2.00 \pm 0.00$ \\
\hline \multirow{2}{*}{ Micrococcus luteus } & WNPs & $5.83 \pm 0.28$ & $4.16 \pm 0.28$ & $3.00 \pm 0.00$ & $2.16 \pm 0.28$ \\
\hline & RNPs & $4.33 \pm 0.57$ & $3.00 \pm 0.00$ & $2.00 \pm 0.00$ & $1.83 \pm 0.28$ \\
\hline
\end{tabular}

Table 2: ZOI (mean \pm SD) observed against various gram-negative bacteria by different concentrations of AgNPs (WNPs \& RNPs).

\begin{tabular}{|c|c|c|c|c|c|}
\hline \multirow{2}{*}{ Bacteria } & & \multicolumn{4}{|c|}{ ZOI (mm) } \\
\hline & & $2.0 \mathrm{mg} / \mathrm{ml}$ & $1.0 \mathrm{mg} / \mathrm{ml}$ & $0.5 \mathrm{mg} / \mathrm{ml}$ & $0.25 \mathrm{mg} / \mathrm{ml}$ \\
\hline \multirow{2}{*}{ Klebsiella pneumoniae } & WNPs & $8.83 \pm 0.28$ & $6.66 \pm 0.28$ & $4.33 \pm 0.57$ & $4.00 \pm 0.00$ \\
\hline & RNPs & $6.83 \pm 0.28$ & $5.16 \pm 0.28$ & $4.00 \pm 0.00$ & $3.33 \pm 0.57$ \\
\hline \multirow{2}{*}{ Salmonella typhimurium } & WNPs & $8.16 \pm 0.28$ & $6.33 \pm 0.57$ & $5.00 \pm 0.00$ & $4.16 \pm 0.28$ \\
\hline & RNPs & $6.33 \pm 0.57$ & $4.83 \pm 0.28$ & $4.00 \pm 0.00$ & $3.16 \pm 0.28$ \\
\hline \multirow{2}{*}{ Enterobacter aerogenes } & WNPs & $5.00 \pm 0.00$ & $3.83 \pm 0.28$ & $2.16 \pm 0.28$ & $2.00 \pm 0.00$ \\
\hline & RNPs & $4.83 \pm 0.28$ & $4.16 \pm 0.28$ & $3.00 \pm 0.00$ & $2.00 \pm 0.00$ \\
\hline \multirow{2}{*}{ Escherichia coli } & WNPs & $5.83 \pm 0.28$ & $4.33 \pm 0.57$ & $3.16 \pm 0.28$ & $3.00 \pm 0.00$ \\
\hline & RNPs & $5.16 \pm 0.28$ & $4.00 \pm 0.00$ & $2.00 \pm 0.00$ & $1.00 \pm 0.00$ \\
\hline \multirow{2}{*}{ Vibrio cholerae } & WNPs & $5.16 \pm 0.28$ & $3.33 \pm 0.57$ & $2.00 \pm 0.00$ & $1.16 \pm 0.28$ \\
\hline & RNPs & $4.33 \pm 0.57$ & $3.00 \pm 0.00$ & $2.16 \pm 0.28$ & $1.00 \pm 0.00$ \\
\hline
\end{tabular}

diffusion results, it was observed that the small-sized WNPs (19.11 $\mathrm{nm})$ generally possessed a more powerful bactericidal effect against both types of bacteria than large-sized RNPs $(33.29 \mathrm{~nm})$. Furthermore, both WNPs and RNPs exhibited a relatively more pronounced bactericidal effect against Gram-negative bacterial strains compared to Gram-positive strains.

The agar-well diffusion method has certain limitations. Agar gel produces hindrance in NPs movement through a solidified gel and affects the releasing of silver ions $\left(\mathrm{Ag}^{+}\right)$from NPs and their contact with bacteria in the surrounding environment $[12,40]$. To overcome these limitations of the agar-gel method, we carried out the XTT salt-based colorimetric method to reconfirm the bactericidal effect of WNPs and RNPs [25-27].

The results obtained in the XTT colorimetric assay demonstrated that at a concentration of $500 \mu \mathrm{g} / \mathrm{ml}$, both WNPs and RNPs showed a complete inhibitory effect against all selected Gram-positive bacteria (Tables 3 and 5). WNPs at a concentration of $250 \mu \mathrm{g} /$ $\mathrm{ml}$ still possessed complete inhibitory effect against $S$. aureus, $S$. mutans, M. luteus, and S. epidermidis. Furthermore, S. mutans was the most sensitive to Gram-positive bacteria to WNPs showing complete inhibition up to the concentration of $125 \mu \mathrm{g} / \mathrm{ml}$. A lowlevel bactericidal effect was observed against $S$. mutans $(14.14 \%)$ and $M$. luteus $(10.33 \%)$ at the concentration of $15.62 \mu \mathrm{g} / \mathrm{ml}$, whereas $7.81 \mu \mathrm{g} / \mathrm{ml}$ was the last concentration up to which $S$. aureus $(11.02 \%)$, S. pneumoniae $(32.60 \%)$, and S. epidermidis (23.0\%) showed bacterial growth inhibition (Table 5). In the case of RNPs-mediated inhibition against Gram-positive bacteria, at a concentration $250 \mu \mathrm{g} / \mathrm{ml}$, S. epidermidis showed complete inhibition, followed by S. aureus $(97.26 \%)>$ M. luteus $(94.63 \%)$ $>$ S. mutans $(89.46 \%)>$ S. pneumoniae $(81.42 \%)$. The last dilution up to which RNPs were found effective against $S$. aureus $(9.29 \%)$, S. pneumoniae $(16.58 \%)$, and S. epidermidis $(33.12 \%)$ was $15.62 \mu \mathrm{g} / \mathrm{ml}$, whereas M. luteus $(9.89 \%)$ and S. mutans $(24.66 \%)$ were inhibited up to the concentration of 7.81 and $31.25 \mu \mathrm{g} / \mathrm{ml}$, respectively (Table 5).

In the case of Gram-negative bacteria, at the concentration of $250 \mu \mathrm{g} / \mathrm{ml}$ of WNPs, except E. coli $(92.73 \%)$, all other bacteria showed complete inhibition (Tables 4 and 6). K. pneumoniae and $S$. typhimurium showed complete inhibition up to the 
Table 3: Measurement (O.D) of growth of different gram-positive bacteria at different concentrations ( $\mu \mathrm{g} / \mathrm{ml})$ of WNPs \& RNPs.

\begin{tabular}{|c|c|c|c|c|c|c|c|c|c|c|c|c|}
\hline Bacteria & & PC & $\mathrm{NC}$ & 500 & 250 & 125 & 62.5 & 31.25 & 15.62 & 7.81 & 3.90 & 1.95 \\
\hline \multirow[b]{2}{*}{ Staphylococcus aureus } & WNPs & $\begin{array}{l}0.069 \pm \\
0.025\end{array}$ & $\begin{array}{c}0.790 \pm \\
0.018\end{array}$ & $\begin{array}{c}0.004 \pm \\
0.004\end{array}$ & $\begin{array}{c}0.007 \pm \\
0.004\end{array}$ & $\begin{array}{l}0.088 \pm \\
0.028\end{array}$ & $\begin{array}{c}0.198 \pm \\
0.034\end{array}$ & $\begin{array}{c}0.314 \pm \\
0.017\end{array}$ & $\begin{array}{l}0.508 \pm \\
0.078\end{array}$ & $\begin{array}{c}0.703 \pm \\
0.061\end{array}$ & $\begin{array}{l}0.738 \pm \\
0.052\end{array}$ & $\begin{array}{l}0.916 \pm \\
0.023\end{array}$ \\
\hline & RNPs & $\begin{array}{c}0.065 \pm \\
0.020\end{array}$ & $\begin{array}{l}0.657 \pm \\
0.015\end{array}$ & $\begin{array}{c}0.008 \pm \\
0.001\end{array}$ & $\begin{array}{l}0.018 \pm \\
0.005\end{array}$ & $\begin{array}{c}0.133 \pm \\
0.032\end{array}$ & $\begin{array}{c}0.337 \pm \\
0.041\end{array}$ & $\begin{array}{l}0.410 \pm \\
0.029\end{array}$ & $\begin{array}{c}0.596 \pm \\
0.056\end{array}$ & $\mathrm{NE}$ & $\mathrm{NE}$ & $\mathrm{NE}$ \\
\hline \multirow{2}{*}{$\begin{array}{c}\text { Streptococcus } \\
\text { pneumoniae }\end{array}$} & WNPs & $\begin{array}{c}0.050 \pm \\
0.031\end{array}$ & $\begin{array}{c}0.865 \pm \\
0.077\end{array}$ & $\begin{array}{c}0.008 \pm \\
0.005\end{array}$ & $\begin{array}{c}0.046 \pm \\
0.025\end{array}$ & $\begin{array}{c}0.098 \pm \\
0.030\end{array}$ & $\begin{array}{c}0.076 \pm \\
0.035\end{array}$ & $\begin{array}{c}0.131 \pm \\
0.033\end{array}$ & $\begin{array}{c}0.260 \pm \\
0.062\end{array}$ & $\begin{array}{c}0.583 \pm \\
0.047\end{array}$ & $\begin{array}{c}0.822 \pm \\
0.031\end{array}$ & $\mathrm{NE}$ \\
\hline & RNPs & $\begin{array}{c}0.049 \pm \\
0.016\end{array}$ & $\begin{array}{c}0.856 \pm \\
0.018\end{array}$ & $\begin{array}{c}0.006 \pm \\
0.003\end{array}$ & $\begin{array}{c}0.159 \pm \\
0.037\end{array}$ & $\begin{array}{c}0.397 \pm \\
0.031\end{array}$ & $\begin{array}{c}0.548 \pm \\
0.037\end{array}$ & $\begin{array}{c}0.653 \pm \\
0.056\end{array}$ & $\begin{array}{c}0.714 \pm \\
0.037\end{array}$ & $\mathrm{NE}$ & $\mathrm{NE}$ & $\mathrm{NE}$ \\
\hline \multirow{2}{*}{$\begin{array}{l}\text { Staphylococcus } \\
\text { epidermidis }\end{array}$} & WNPs & $\begin{array}{c}0.112 \pm \\
0.039\end{array}$ & $\begin{array}{l}0.917 \pm \\
0.045\end{array}$ & $\begin{array}{c}0.007 \pm \\
0.004\end{array}$ & $\begin{array}{c}0.009 \pm \\
0.006\end{array}$ & $\begin{array}{l}0.065 \pm \\
0.025\end{array}$ & $\begin{array}{c}0.168 \pm \\
0.043\end{array}$ & $\begin{array}{c}0.275 \pm \\
0.046\end{array}$ & $\begin{array}{c}0.479 \pm \\
0.030\end{array}$ & $\begin{array}{l}0.706 \pm \\
0.023\end{array}$ & $\begin{array}{c}0.877 \pm \\
0.031\end{array}$ & $\mathrm{NE}$ \\
\hline & RNPs & $\begin{array}{c}0.073 \pm \\
0.017\end{array}$ & $\begin{array}{c}0.809 \pm \\
0.054\end{array}$ & $\begin{array}{l}0.009 \pm \\
0.003\end{array}$ & $\begin{array}{c}0.008 \pm \\
0.001\end{array}$ & $\begin{array}{c}0.022 \pm \\
0.011\end{array}$ & $\begin{array}{c}0.096 \pm \\
0.010\end{array}$ & $\begin{array}{c}0.264 \pm \\
0.054\end{array}$ & $\begin{array}{c}0.541 \pm \\
0.064\end{array}$ & $\begin{array}{c}0.799 \pm \\
0.087\end{array}$ & $\mathrm{NE}$ & $\mathrm{NE}$ \\
\hline \multirow{2}{*}{ Streptococcus mutans } & WNPs & $\begin{array}{c}0.077 \pm \\
0.038\end{array}$ & $\begin{array}{l}0.728 \pm \\
0.060\end{array}$ & $\begin{array}{l}0.006 \pm \\
0.002\end{array}$ & $\begin{array}{l}0.003 \pm \\
0.003\end{array}$ & $\begin{array}{l}0.009 \pm \\
0.006\end{array}$ & $\begin{array}{l}0.090 \pm \\
0.015\end{array}$ & $\begin{array}{l}0.201 \pm \\
0.030\end{array}$ & $\begin{array}{l}0.625 \pm \\
0.065\end{array}$ & $\mathrm{NE}$ & $\mathrm{NE}$ & NE \\
\hline & RNPs & $\begin{array}{c}0.082 \pm \\
0.013\end{array}$ & $\begin{array}{l}0.750 \pm \\
0.035\end{array}$ & $\begin{array}{c}0.004 \pm \\
0.001\end{array}$ & $\begin{array}{c}0.079 \pm \\
0.011\end{array}$ & $\begin{array}{c}0.257 \pm \\
0.022\end{array}$ & $\begin{array}{c}0.451 \pm \\
0.033\end{array}$ & $\begin{array}{c}0.565 \pm \\
0.062\end{array}$ & $\mathrm{NE}$ & NE & $\mathrm{NE}$ & $\mathrm{NE}$ \\
\hline \multirow{2}{*}{ Micrococcus luteus } & WNPs & $\begin{array}{c}0.045 \pm \\
0.028\end{array}$ & $\begin{array}{c}0.842 \pm \\
0.062\end{array}$ & $\begin{array}{l}0.006 \pm \\
0.003\end{array}$ & $\begin{array}{c}0.004 \pm \\
0.004\end{array}$ & $\begin{array}{l}0.158 \pm \\
0.042\end{array}$ & $\begin{array}{l}0.408 \pm \\
0.033\end{array}$ & $\begin{array}{c}0.662 \pm \\
0.037\end{array}$ & $\begin{array}{c}0.755 \pm \\
0.046\end{array}$ & $\mathrm{NE}$ & $\mathrm{NE}$ & $\mathrm{NE}$ \\
\hline & RNPs & $\begin{array}{l}0.076 \pm \\
0.006\end{array}$ & $\begin{array}{l}0.708 \pm \\
0.023\end{array}$ & $\begin{array}{c}0.007 \pm \\
0.002\end{array}$ & $\begin{array}{c}0.038 \pm \\
0.004\end{array}$ & $\begin{array}{c}0.078 \pm \\
0.006\end{array}$ & $\begin{array}{c}0.176 \pm \\
0.013\end{array}$ & $\begin{array}{c}0.372 \pm \\
0.007\end{array}$ & $\begin{array}{c}0.520 \pm \\
0.040\end{array}$ & $\begin{array}{c}0.638 \pm \\
0.028\end{array}$ & $\mathrm{NE}$ & $\mathrm{NE}$ \\
\hline
\end{tabular}

PC: positive control; NC: negative control; NE: No significant bactericidal effect.

Table 4: Measurement (O.D) of growth of different gram-negative bacteria at different concentrations of ( $\mu \mathrm{g} / \mathrm{ml}) \mathrm{WNPs} \& \mathrm{RNPs}$.

\begin{tabular}{|c|c|c|c|c|c|c|c|c|c|c|c|c|}
\hline Bacteria & & PC & $\mathrm{NC}$ & 500 & 250 & 125 & 62.5 & 31.25 & 15.62 & 7.81 & 3.90 & 1.95 \\
\hline \multirow{2}{*}{$\begin{array}{c}\text { Klebsiella } \\
\text { pneumoniae }\end{array}$} & WNPs & $\begin{array}{c}0.101 \pm \\
0.029\end{array}$ & $\begin{array}{c}0.850 \pm \\
0.050\end{array}$ & $\begin{array}{c}0.004 \pm \\
0.003\end{array}$ & $\begin{array}{l}0.006 \pm \\
0.003\end{array}$ & $\begin{array}{l}0.006 \pm \\
0.005\end{array}$ & $\begin{array}{c}0.139 \pm \\
0.026\end{array}$ & $\begin{array}{c}0.384 \pm \\
0.027\end{array}$ & $\begin{array}{c}0.596 \pm \\
0.012\end{array}$ & $\begin{array}{c}0.649 \pm \\
0.053\end{array}$ & $\begin{array}{c}0.784 \pm \\
0.066\end{array}$ & $\begin{array}{c}0.968 \pm \\
0.038\end{array}$ \\
\hline & RNPs & $\begin{array}{c}0.057 \pm \\
0.016\end{array}$ & $\begin{array}{c}0.805 \pm \\
0.064\end{array}$ & $\begin{array}{c}0.007 \pm \\
0.001\end{array}$ & $\begin{array}{c}0.006 \pm \\
0.002\end{array}$ & $\begin{array}{c}0.080 \pm \\
0.012\end{array}$ & $\begin{array}{c}0.207 \pm \\
0.023\end{array}$ & $\begin{array}{c}0.351 \pm \\
0.034\end{array}$ & $\begin{array}{c}0.498 \pm \\
0.019\end{array}$ & $\begin{array}{c}0.657 \pm \\
0.052\end{array}$ & $\begin{array}{c}0.839 \pm \\
0.019\end{array}$ & $\begin{array}{c}0.902 \pm \\
0.023\end{array}$ \\
\hline \multirow{2}{*}{$\begin{array}{c}\text { Salmonella } \\
\text { typhimurium }\end{array}$} & WNPs & $\begin{array}{c}0.055 \pm \\
0.024\end{array}$ & $\begin{array}{c}0.834 \pm \\
0.024\end{array}$ & $\begin{array}{c}0.007 \pm \\
0.001\end{array}$ & $\begin{array}{l}0.005 \pm \\
0.003\end{array}$ & $\begin{array}{c}0.009 \pm \\
0.001\end{array}$ & $\begin{array}{c}0.112 \pm \\
0.017\end{array}$ & $\begin{array}{c}0.443 \pm \\
0.036\end{array}$ & $\begin{array}{c}0.542 \pm \\
0.030\end{array}$ & $\begin{array}{c}0.677 \pm \\
0.024\end{array}$ & $\begin{array}{c}0.767 \pm \\
0.043\end{array}$ & $\mathrm{NE}$ \\
\hline & RNPs & $\begin{array}{c}0.076 \pm \\
0.016\end{array}$ & $\begin{array}{c}0.722 \pm \\
0.038\end{array}$ & $\begin{array}{c}0.004 \pm \\
0.001\end{array}$ & $\begin{array}{c}0.016 \pm \\
0.003\end{array}$ & $\begin{array}{c}0.052 \pm \\
0.014\end{array}$ & $\begin{array}{c}0.101 \pm \\
0.016\end{array}$ & $\begin{array}{c}0.393 \pm \\
0.020\end{array}$ & $\begin{array}{c}0.574 \pm \\
0.050\end{array}$ & $\mathrm{NE}$ & $\mathrm{NE}$ & $\mathrm{NE}$ \\
\hline \multirow{2}{*}{$\begin{array}{c}\text { Enterobacter } \\
\text { aerogenes }\end{array}$} & WNPs & $\begin{array}{c}0.114 \pm \\
0.038\end{array}$ & $\begin{array}{c}0.774 \pm \\
0.039\end{array}$ & $\begin{array}{c}0.004 \pm \\
0.004\end{array}$ & $\begin{array}{c}0.005 \pm \\
0.003\end{array}$ & $\begin{array}{c}0.097 \pm \\
0.031\end{array}$ & $\begin{array}{c}0.184 \pm \\
0.037\end{array}$ & $\begin{array}{c}0.293 \pm \\
0.088\end{array}$ & $\begin{array}{c}0.337 \pm \\
0.044\end{array}$ & $\begin{array}{c}0.524 \pm \\
0.108\end{array}$ & $\mathrm{NE}$ & $\mathrm{NE}$ \\
\hline & RNPs & $\begin{array}{c}0.105 \pm \\
0.009\end{array}$ & $\begin{array}{c}0.728 \pm \\
0.018\end{array}$ & $\begin{array}{c}0.004 \pm \\
0.002\end{array}$ & $\begin{array}{c}0.059 \pm \\
0.016\end{array}$ & $\begin{array}{c}0.065 \pm \\
0.022\end{array}$ & $\begin{array}{c}0.165 \pm \\
0.032\end{array}$ & $\begin{array}{c}0.248 \pm \\
0.033\end{array}$ & $\begin{array}{c}0.425 \pm \\
0.028\end{array}$ & $\begin{array}{c}0.698 \pm \\
0.022\end{array}$ & $\mathrm{NE}$ & $\mathrm{NE}$ \\
\hline \multirow{2}{*}{ Escherichia coli } & WNPs & $\begin{array}{c}0.035 \pm \\
0.007\end{array}$ & $\begin{array}{c}0.908 \pm \\
0.044\end{array}$ & $\begin{array}{c}0.006 \pm \\
0.003\end{array}$ & $\begin{array}{c}0.066 \pm \\
0.024\end{array}$ & $\begin{array}{c}0.091 \pm \\
0.014\end{array}$ & $\begin{array}{c}0.138 \pm \\
0.023\end{array}$ & $\begin{array}{c}0.170 \pm \\
0.035\end{array}$ & $\begin{array}{c}0.399 \pm \\
0.023\end{array}$ & $\begin{array}{c}0.619 \pm \\
0.035\end{array}$ & $\begin{array}{c}0.814 \pm \\
0.045\end{array}$ & $\begin{array}{c}0.985 \pm \\
0.007\end{array}$ \\
\hline & RNPs & $\begin{array}{c}0.088 \pm \\
0.010\end{array}$ & $\begin{array}{c}0.685 \pm \\
0.027\end{array}$ & $\begin{array}{c}0.005 \pm \\
0.002\end{array}$ & $\begin{array}{c}0.028 \pm \\
0.001\end{array}$ & $\begin{array}{c}0.116 \pm \\
0.023\end{array}$ & $\begin{array}{c}0.289 \pm \\
0.019\end{array}$ & $\begin{array}{c}0.404 \pm \\
0.009\end{array}$ & $\begin{array}{c}0.597 \pm \\
0.033\end{array}$ & $\begin{array}{c}0.729 \pm \\
0.024\end{array}$ & $\begin{array}{c}0.795 \pm \\
0.016\end{array}$ & $\begin{array}{c}0.703 \pm \\
0.014\end{array}$ \\
\hline \multirow{2}{*}{ Vibrio cholerae } & WNPs & $\begin{array}{c}0.086 \pm \\
0.005\end{array}$ & $\begin{array}{c}0.766 \pm \\
0.071\end{array}$ & $\begin{array}{c}0.006 \pm \\
0.001\end{array}$ & $\begin{array}{c}0.005 \pm \\
0.001\end{array}$ & $\begin{array}{c}0.076 \pm \\
0.014\end{array}$ & $\begin{array}{c}0.093 \pm \\
0.008\end{array}$ & $\begin{array}{c}0.168 \pm \\
0.021\end{array}$ & $\begin{array}{c}0.345 \pm \\
0.048\end{array}$ & $\begin{array}{c}0.562 \pm \\
0.053\end{array}$ & $\mathrm{NE}$ & $\mathrm{NE}$ \\
\hline & RNPs & $\begin{array}{c}0.092 \pm \\
0.004\end{array}$ & $\begin{array}{c}0.698 \pm \\
0.049\end{array}$ & $\begin{array}{c}0.006 \pm \\
0.001\end{array}$ & $\begin{array}{c}0.086 \pm \\
0.025\end{array}$ & $\begin{array}{c}0.179 \pm \\
0.022\end{array}$ & $\begin{array}{c}0.382 \pm \\
0.027\end{array}$ & $\begin{array}{c}0.565 \pm \\
0.059\end{array}$ & $\mathrm{NE}$ & $\mathrm{NE}$ & $\mathrm{NE}$ & $\mathrm{NE}$ \\
\hline
\end{tabular}

PC: Positive control; NC: Negative control; NE: No significant bactericidal effect.

concentration of $125 \mu \mathrm{g} / \mathrm{ml}$, followed by $V$. cholerae $(90.07 \%)$, E. coli $(89.97 \%)$, and E. aerogenes $(87.46 \%)$. WNPs up to the concentration of $7.81 \mu \mathrm{g} / \mathrm{ml}$ displayed a moderate level inhibitory effect in the order of E. aerogenes $(32.29 \%)>$ E. coli $(31.82 \%)>V$. cholerae $(26.63 \%)>K$. pneumoniae $(23.64 \%)>$
S. typhimurium (18.82\%). Similarly, RNPs at the concentration of $250 \mu \mathrm{g} / \mathrm{ml}$ demonstrated complete inhibition of $K$. pneumoniae, followed by S. typhimurium $(97.78 \%)>$ E. coli $(95.91 \%)>E$. aerogenes $(91.89 \%)$, and least effective against $V$. cholerae $(87.67 \%)$. In the case of RNPs, a moderate level of bactericidal 
Table 5: Observation of percent reduction (\%) in bacterial growth in the presence of different concentrations ( $\mu \mathrm{g} / \mathrm{ml})$ of WNPs \& RNPs.

\begin{tabular}{|c|c|c|c|c|c|c|c|c|c|c|c|}
\hline Bacteria & & PC & 500 & 250 & 125 & 62.5 & 31.25 & 15.62 & 7.81 & 3.90 & 1.95 \\
\hline \multirow{2}{*}{$\begin{array}{c}\text { Staphylococcu } \\
\text { aureus }\end{array}$} & WNPs & 91.26 & $\mathrm{CI}$ & CI & 88.86 & 74.93 & 60.25 & 35.69 & 11.02 & +6.58 & +15.94 \\
\hline & RNPS & 90.10 & $\mathrm{CI}$ & 97.26 & 79.75 & 48.70 & 37.59 & 9.29 & NE & $\mathrm{NE}$ & $\mathrm{NE}$ \\
\hline \multirow{2}{*}{$\begin{array}{l}\text { Streptococcus } \\
\text { pneumoniae }\end{array}$} & WNPs & 94.21 & $\mathrm{CI}$ & 94.68 & 88.67 & 91.21 & 84.85 & 69.94 & 32.60 & 4.97 & $\mathrm{NE}$ \\
\hline & RNPS & 94.27 & $\mathrm{CI}$ & 81.42 & 53.62 & 35.98 & 23.71 & 16.58 & NE & $\mathrm{NE}$ & $\mathrm{NE}$ \\
\hline \multirow{2}{*}{$\begin{array}{l}\text { Staphylococcus } \\
\text { epidermidis }\end{array}$} & WNPs & 87.78 & $\mathrm{CI}$ & CI & 92.91 & 81.67 & 70.01 & 47.76 & 23.00 & 4.36 & $\mathrm{NE}$ \\
\hline & RNPS & 90.97 & $\mathrm{CI}$ & $\mathrm{CI}$ & 97.28 & 88.13 & 67.36 & 33.12 & 1.23 & NE & $\mathrm{NE}$ \\
\hline \multirow{2}{*}{$\begin{array}{c}\text { Streptococcus } \\
\text { mutans }\end{array}$} & WNPs & 89.42 & CI & CI & CI & 87.63 & 72.39 & 14.14 & NE & $\mathrm{NE}$ & $\mathrm{NE}$ \\
\hline & RNPS & 89.06 & $\mathrm{CI}$ & 89.46 & 65.73 & 39.86 & 24.66 & $\mathrm{NE}$ & $\mathrm{NE}$ & NE & $\mathrm{NE}$ \\
\hline \multirow{2}{*}{ Micrococcus luteus } & WNPs & 94.65 & $\mathrm{CI}$ & CI & 81.23 & 51.54 & 21.37 & 10.33 & $\mathrm{NE}$ & $\mathrm{NE}$ & $\mathrm{NE}$ \\
\hline & RNPS & 89.26 & $\mathrm{CI}$ & 94.63 & 88.98 & 75.14 & 47.45 & 26.55 & 9.89 & NE & $\mathrm{NE}$ \\
\hline
\end{tabular}

NE: No significant bactericidal effect; CI: Complete inhibition (reduction $>99.0 \%$ ).

Table 6: Observation of percent reduction (\%) in bacterial growth in the presence of different concentrations ( $\mu \mathrm{g} / \mathrm{ml})$ of WNPs \& RNPs.

\begin{tabular}{|c|c|c|c|c|c|c|c|c|c|c|c|}
\hline Bacteria & & PC & 500 & 250 & 125 & 62.5 & 31.25 & 15.62 & 7.81 & 3.90 & 1.95 \\
\hline \multirow{2}{*}{$\begin{array}{c}\text { Klebsiella } \\
\text { pneumoniae }\end{array}$} & WNPs & 88.11 & CI & CI & $\mathrm{CI}$ & 83.64 & 54.82 & 29.88 & 23.64 & 7.77 & +11.38 \\
\hline & RNPS & 92.91 & CI & CI & 90.06 & 74.28 & 56.39 & 38.13 & 18.38 & +4.22 & +12.04 \\
\hline \multirow{2}{*}{$\begin{array}{l}\text { Salmonella } \\
\text { typhimurium }\end{array}$} & WNPs & 93.40 & CI & $\mathrm{CI}$ & $\mathrm{CI}$ & 86.57 & 46.88 & 35.01 & 18.82 & 8.03 & $\mathrm{NE}$ \\
\hline & RNPS & 89.47 & $\mathrm{CI}$ & 97.78 & 92.79 & 86.01 & 45.56 & 20.49 & $\mathrm{NE}$ & $\mathrm{NE}$ & $\mathrm{NE}$ \\
\hline \multirow{2}{*}{$\begin{array}{c}\text { Enterobacter } \\
\text { aerogenes }\end{array}$} & WNPs & 85.27 & $\mathrm{CI}$ & CI & 87.46 & 76.22 & 62.14 & 56.45 & 32.29 & $\mathrm{NE}$ & $\mathrm{NE}$ \\
\hline & RNPS & 85.57 & $\mathrm{CI}$ & 91.89 & 91.07 & 77.33 & 65.93 & 41.62 & 4.12 & $\mathrm{NE}$ & $\mathrm{NE}$ \\
\hline \multirow{2}{*}{ Escherichia coli } & WNPs & 96.14 & CI & 92.73 & 89.97 & 84.80 & 81.27 & 56.05 & 31.82 & 10.35 & +8.48 \\
\hline & RNPS & 87.15 & $\mathrm{CI}$ & 95.91 & 83.06 & 57.81 & 41.02 & 12.84 & +6.42 & +16.05 & +2.62 \\
\hline \multirow{2}{*}{ Vibrio cholerae } & WNPs & 88.77 & CI & CI & 90.07 & 87.85 & 78.06 & 54.96 & 26.63 & $\mathrm{NE}$ & $\mathrm{NE}$ \\
\hline & RNPS & 86.81 & CI & 87.67 & 74.35 & 45.27 & 19.05 & $\mathrm{NE}$ & $\mathrm{NE}$ & $\mathrm{NE}$ & NE \\
\hline
\end{tabular}

NE: No significant bactericidal effect; CI: Complete inhibition (reduction $>99.0 \%$ ).

activity was observed up to the concentration of $15.62 \mu \mathrm{g} / \mathrm{ml}$ against $E$. aerogenes $(41.62 \%)$ and $K$. pneumoniae $(38.13 \%)$, and low-level bactericidal effect against S. typhimurium $(20.49 \%)$ and E. coli (12.84\%), as shown in Table 6.

MIC and MBC concentrations were estimated against all the experimental bacteria in the presence of different concentrations of both WNPs and RNPs. The results revealed that both MIC and MBC (WNPs) values were estimated to $125 \mu \mathrm{g} / \mathrm{ml}$ for bacteria $S$. mutans, $K$. pneumoniae, and $S$. typhimurium, $250 \mu \mathrm{g} / \mathrm{ml}$ for $S$. aureus, $M$. luteus, and $S$. epidermidis and 500 $\mu \mathrm{g} / \mathrm{ml}$ for $E$. coli. In the case of $S$. pneumoniae, E. aerogenes, and $V$. cholerae, MIC and MBC values were observed at 250 and $500 \mu \mathrm{g} / \mathrm{ml}$, respectively. Furthermore, in the case of RNPsmediated inhibition, both MIC and MBC values were found at $250 \mu \mathrm{g} / \mathrm{ml}$ for S. epidermidis and K. pneumoniae and $500 \mu \mathrm{g} / \mathrm{ml}$ in case of all other bacterial strains.
In the present study, besides observation of concentrationdependent bactericidal efficiency of both AgNPs, we also observed that WNPs-treated $S$. aureus showed positive growth (growth more than the negative control) at $3.90 \mu \mathrm{g}$ / $\mathrm{ml}(+6.58 \%)$ and $1.95 \mu \mathrm{g} / \mathrm{ml}(15.94 \%)$ concentrations (Table 5). WNPs also exhibited positive growth with respect to Gram-negative bacteria $K$. pneumoniae $(+11.38 \%)$ and E. coli $(+8.48 \%)$ at the concentration of $1.95 \mu \mathrm{g} / \mathrm{ml}$ (Table 6$)$. In the case of RNP-mediated inhibition, K. pneumoniae demonstrated increased positive growth from $4.22 \%$ to $12.04 \%$ with respect to a decrease concentration of AgNPs from 3.90 to $1.95 \mu \mathrm{g} / \mathrm{ml}$. Similarly, from concentrations 7.81 to $3.90 \mu \mathrm{g} / \mathrm{ml}$, RNPs-treated E. coli demonstrated an increase in positive growth from $6.42 \%$ to $16.05 \%$ (Table 6 ). This positive growth was not observed in further dilutions $(<1.95 \mu \mathrm{g} / \mathrm{ml})$ in any case. The low dose of WNPs, which stimulated the positive growth of $S$. aureus 
was found $0.78 \%(1.95 \mu \mathrm{g} / \mathrm{ml})$ and $1.56 \%(3.90 \mu \mathrm{g} / \mathrm{ml})$ of MIC value. In the case of Gram-negative bacteria K. pneumoniae and E. coli, WNPs enhancer dose was found $1.56 \%$ and $0.39 \%$ of their individual MIC values, respectively. Furthermore, a low dose of RNPs, which boosted the growth, was observed to be $0.78 \%-1.56 \%$ of MIC values of both $E$. coli and $K$. pneumoniae.

Several research groups elucidated that AgNPs may inhibit bacterial growth or cell lysis via multiple mechanisms such as adhesion on the bacterial surface through electrostatic attraction between AgNPs and bacterial membrane residues, which directly hampered the permeation of essential nutrients inside the cell $[9,41]$, or penetration through the membrane by making holes, or by altering the structural integrity and disturbing the osmoregulation of bacterial cell $[8,42]$. Inside the cell, AgNPs release $\mathrm{Ag}+$ ions which directly generate reactive oxygen species (ROS), and these species may inactivate respiratory enzymes, or may inhibit bacterial replication, transcription or translation and create hindrance in the expression of cellular proteins which ultimately cause bacterial death $[9,11,16]$. The reason for the bactericidal effect of AgNPs is multifactorial and requires the synergistic effect of all or any combination of the above-mentioned pathways.

Recently, plants extract-based synthesis of AgNPs is gaining popularity due to its great inhibitory potential against a high range of bacteria $[10,12,16,29,31,32,35,42]$. For instance, AgNPs synthesized from plants such as M. nigra, C. nucifera, Abutilon indicum, Aloe vera, and $A$. indica were observed to be a potential bactericidal agent against both gram-positive and gram-negative bacteria like $S$. epidermidis, $S$. pneumoniae, S. aureus, E. aerogenes, K. pneumoniae, S. mutans, M. luteus, Salmonella paratyphi, Salmonella typhimurium, V. cholerae, E. coli, P. aeruginosa, etc. $[11,14,18,31,37,43,44]$. Some of the studies have further shown AgNPs' bactericidal effect against multi-drug resistant bacteria such as $P$. aeruginosa, E. coli, Streptococcus pyogens, and $S$. aureus $[14,15,29,45-47]$. In the present study, both WNPs and RNPs elucidated promissory inhibitory potential against all the Gram-positive and Gram-negative experimental bacteria, but the profound bactericidal effect was observed against $S$. epidermidis, $S$. aureus, $S$. mutans, $K$. pneumoniae, and $S$. typhimurium as described earlier in results.

Results from various studies substantiated the fact that the antibacterial effect of AgNPs is dependent on their shape $[48,49]$, size, concentrations, rate of dissolution of silver ions from NPs [28,44,47,50,51], agglomeration tendency of NPs, aerobic and anaerobic conditions, surface modification of AgNPs, and type of microorganisms [12,52]. It is now well documented in the literature that the release of silver ions from larger NPs is slower in comparison to small size NPs [40]. With a decrease in particle size, there is enhancement in surface areato-volume $(\mathrm{S} / \mathrm{V})$ ratio which directly affects the antibacterial potential of AgNPs. In small NPs, bandgap energy increases and excess energy is observed at the surface of particles which is responsible for lattice contractions. Furthermore, these lattice contractions favor the confinement of electrons and enhance their direct interactions with the bacterial cell wall, hence their killings $[8,49,53]$. The present study reinforced this conception as we have also observed that small-sized WNPs (19.11 nm) possessed more profound bactericidal efficiency than largesized RNPs (33.85 nm) against both Gram-negative and Grampositive experimental bacterial strains.

A perusal of literature authenticates the notion that MIC and $\mathrm{MBC}$ values were dependent on the size and dose of AgNPs. Agnihotri et al. [8] revealed that both $\mathrm{MIC}$ and $\mathrm{MBC}$ concentrations were found to be the size and dose-dependent and observed as 20-110 and 30-140 $\mu \mathrm{g} / \mathrm{ml}$ against E. coli, 30-120 and 40-140 $\mu \mathrm{g} / \mathrm{ml}$ for Bacillus subtilis, and $70-200 \mu \mathrm{g} / \mathrm{ml}$ and 80 to $>200$ $\mu \mathrm{g} / \mathrm{ml}$ for $S$. aureus, respectively. It has also been observed in the same study that $20 \mu \mathrm{g} / \mathrm{ml}$ of AgNPs of size $10 \mathrm{~nm}$ caused a $53 \%$ reduction in cell density as compared to control in E. coli and $84 \%$ reduction in case of $B$. subtilis, whereas in the case of $S$. aureus at $80 \mu \mathrm{g} / \mathrm{ml}$ concentration $97 \%$ reduction in bacterial cell density was observed. Similarly, in some other studies, MIC was found to be $100 \mu \mathrm{g} / \mathrm{ml}$ against $S$. aureus and E. coli, 25-100 $\mu \mathrm{g} / \mathrm{ml}$ against E.coli, S. typhi, and $S$. aureus by $10-25 \mathrm{~nm}$-sized AgNPs, $75 \mu \mathrm{g} / \mathrm{ml}$ against E. coli, $V$. cholerae, and $S$. typhi by $21 \mathrm{~nm}$-sized AgNPs [29,54,55]. Tanase et al. [51] demonstrated that AgNPs of an average size of $44 \mathrm{~nm}$ synthesized from Picea abies L. showed MIC in the range of $0.05-0.63 \mathrm{mg} / \mathrm{ml}$ and $\mathrm{MBC}$ in the range of $0.25-1.57 \mathrm{mg} / \mathrm{ml}$ against various bacterial strains including $S$. aureus, E. coli, $K$. pneumoniae, multiple drug-resistant $S$. aureus, and $P$. aeruginosa. In the case of ultrasmall sized AgNPs (4-5.5 nm), two studies reported MIC values in the range of $0.2-7.8 \mu \mathrm{g} / \mathrm{mL}$ against $K$. pneumoniae, E. coli, $S$. aureus, and $S$. typhimurium [44,56]. Recently, Dong et al. [28] also revealed the size-dependent bactericidal effect of AgNPs, whereas AgNPs with size $10 \pm 5 \mathrm{~nm}$ were found to have a lower MIC value (1.0 vs. $11.5 \mu \mathrm{g} / \mathrm{ml})$ in comparison to AgNPs with size $90 \pm 5 \mathrm{~nm}$. In accordance with the previous studies, we have also observed that the antibacterial potential of AgNPs was found to be dose and size-dependent. WNPs (19.11 nm) exhibited MIC and $\mathrm{MBC}$ values in the concentration range of $125-250 \mu \mathrm{g} /$ $\mathrm{ml}$, whereas large-sized RNPs $(33.85 \mathrm{~nm})$ displayed values in the range of $250-500 \mu \mathrm{g} / \mathrm{ml}$. Furthermore, small-sized WNPs showed complete inhibition of $S$. mutans, K. pneumonia, and $S$. typhimurium up to the concentration of $125 \mu \mathrm{g} / \mathrm{ml}$, whereas largesized RNPs showed complete inhibition against these bacterial strains at concentration 250-500 $\mu \mathrm{g} / \mathrm{ml}$ (Tables 5 and 6). These variations in results with comparison to previous studies may be attributed to the differential level of tolerance of bactericidal agents (here AgNPs) by the experimental strains, size, and shape of NPs and different methodology adopted by researchers [29].

During the evaluation of the bactericidal effect of AgNPs, contrary to our expectations, some bacterial strains showed stimulated growth in the medium at certain lower concentrations of AgNPs, as shown in Tables 5 and 6. One Gram-positive bacteria $S$. aureus $(+6.58 \%$ to $+15.94 \%$ ) and two Gram-negative bacteria $K$. pneumoniae $(+11.38 \%)$ and E. coli $(+8.48 \%)$ demonstrated enhanced growth in the presence of low-dose WNPs $(\leq 3.90 \mu \mathrm{g} / \mathrm{ml})$. Similarly, the presence of low-dose RNPs $(<7.81 \mu \mathrm{g} / \mathrm{ml})$ stimulated the growth of $K$. pneumoniae $(+4.22 \%$ to $+11.38 \%)$ and $E$. coli $(+6.42 \%$ to $+16.05 \%$ ), as shown in Table 6. Previously, it was shown that sublethal concentration (3-8 $\mu \mathrm{g} / \mathrm{l})$ of $\mathrm{AgNO}_{3}$ boosted $E$. coli growth, and it was further documented that $\operatorname{Ag}_{ \pm}(<15 \mu \mathrm{g} / \mathrm{l})$ were found to stimulate the E. coli growth instead of its inhibition [40]. Similarly, 
these unexpected results were also reported by a handful of studies. Schacht et al. [57] demonstrated that at certain lower concentrations $(20-60 \mu \mathrm{g} / \mathrm{ml})$ of AgNPs ( $<15 \mathrm{~nm})$, Cupriavidus necator exhibited stimulated growth. Xiu et al. [40] reported that polyethyleneglycol (2.8-10.5 nm) and polyvinyl pyrrolidone $(20-80 \mathrm{~nm})$ coated AgNPs enhanced the E. coli K12 growth from $6 \%$ to $13 \%$ (at a concentration of $1.8-2.2 \mu \mathrm{g} / \mathrm{ml}$ ) and $11-21 \%$ (at a concentration of 5.7-16.4 $\mu \mathrm{g} / \mathrm{ml}$ ), respectively. Similarly, Polyvinylpyrrolidone (PVP)-coated AgNPs (27.1 nm) were observed to boost the growth of $E$. coli up to $29.9 \%$ at the concentration of $0.34 \mu \mathrm{g} / \mathrm{ml}[58,59]$. Not only AgNPs, the stimulated growth was also observed with zinc NPs, where at certain sub-lethal concentrations, zinc NPs of size 90-100 nm escalated the biofilm formation capacity of $V$. cholerae [18]. Moreover, Rodrigues et al. [60] demonstrated that single-walled carbon nanotubes increase bacterial cell growth and biofilm formation in $E$. coli $\mathrm{K} 12$ bacteria at a concentration between 5 and $300 \mu \mathrm{g} / \mathrm{ml}$. In the present study, sub-lethal concentrations that enhanced the growth of bacteria were found in the range of $1.95-7.81 \mu \mathrm{g} / \mathrm{ml}$ against bacteria $S$. aureus, K. pneumoniae, and $E$. coli and these low-dose concentrations were found to be $0.39 \%$ $1.56 \%$ of individual MIC value of the respective bacterial strains, as explained in the result section.

This stimulated growth in the presence of certain lower concentrations (sub-lethal) of inhibitors is not a new phenomenon in microbes and is known as hormetic response, which is a biphasic dose-response to an inhibitory agent and is defined by a high dose inhibitory and a low dose stimulatory effect on the growth of microbes $[61,62]$. This response was reported in many studies where antibiotic stress was tolerated, and a new way of survival was achieved, which was found to be responsible for exponential growth $[62,63]$. Similarly, NPs exposure and its relation with hormetic response have been studied [59], although the exact mechanism of this altered behavior of the bacterial cell is not defined. By perusal of recent literature, we tried to explain one of the possible mechanisms behind this unusual "enhanced growth", which may be due to a non-heritable phenomenon called "persistence". Persister cells are those sets of subpopulation cells that are killed by bactericidal agents much slower than other subpopulation cells sets [64]. The bactericidal effect of AgNPs is generally triggered by the dissolution of the crystalline core of AgNPs and the release of soluble silver ions in the medium [40]. Positively charged silver ions $\left(\mathrm{Ag}^{+}\right)$damage DNA inside the bacterial cell by interacting with negatively charged phosphate components of DNA and also by overproducing reactive oxygen (ROS) species [12,35]. In the presence of a high amount of $\mathrm{Ag}^{+}$, the bacterial defense mechanism failed to eliminate the ROS species, which are overproduced by the AgNPs-mediated stress. Finally, ROS destroy the redox homeostasis of bacteria and cause lipid peroxidation of cell membrane along with damage to replication, transcriptional and translational machinery, which ultimately resulted in bacterial death $[12,47]$. But in the presence of a low level of AgNPs $\left(\mathrm{Ag}^{+}\right)$concentration, the bacterial defense system has time to respond to AgNPs-mediated toxicity. In this case, due to AgNPs-mediated genotoxicity, there is an accumulation of the aberrant amount of ssDNA inside the bacterial cell, which induces save our souls (SOS) response, a response by DNA-repair regulatory network $[28,47]$. Furthermore, this continues AgNPsmediated toxicity produce selection pressure through "DNA repair heterogeneity" in a certain population of bacteria, and this resulted in the formation of subpopulations of susceptible and persistent cells [64]. This "DNA repair heterogeneity" is a stressacquired characteristic that is achieved either by mutating and rearrangement of their genome or by differential regulation of genes like DNA-dependent kinase [12]. So, in the case of low-dose (sub-lethal) concentrations of AgNPs, susceptible cells die (which failed to adapt), and persister cells survive by tolerating sub-lethal dose thorough this stress-acquired adaptation. Furthermore, this adaptation potentiates persister cells' survival and provokes their surge to overcompensate cell damage and population size $[17,65-$ 67]. These homeostatic or compensatory set points were achieved by the involvement of various physiological cellular processes, which coordinately and synergistically produce an enhanced stress resistance capacity along with elevated adaptability of persister cells by increasing their cell growth in media $[61,62,64,68-70]$. This may be one of the probable explanations of hormetic response, which is based on observations made in recent literature data. More studies are required to authenticate such results and to decipher the biochemical or molecular pathways responsible for such stimulated growth.

Global dissemination of antibiotic-resistant pathogenic bacterial strains resulted in increasing cases of mortality and morbidity worldwide [71]. The development of new-generation bactericidal agents requires a complete understanding of bacterial response to different bactericidal agents, and this knowledge can be further exploited for designing future NPsbased antibacterial agents [17,71]. Today's one of the promising methods for environmentally benign production of AgNPs is believed to be those involving reaction mechanisms mediated by low-cost and non-hazardous phytochemicals from the plant kingdom. Plants contain wide varieties of phytochemicals with high reduction potential, which is an essential property for mediating reactions involving reduction of metal salts for final production of non-structural elemental metal NPs $[2,5,10,16]$. Crop plants like wheat and rice are annual crops that are easy to grow and easy to maintain compared to other plants species and, therefore, represent a sustainable alternative for biofabrication of various metallic NPs. This plant-based strategy can mostly remain clean, low energy consumption, easy to scaled-up, highly reproducible, and produces AgNPs with great biocompatibility.

\section{CONCLUSION}

In the present investigation, a green chemistry synthetic route with the help of water extracts of the leaflets of wheat and rice plants was used for the synthesis of well-dispersed AgNPs under controlled processing parameters. These AgNPs were found to be highly effective as a bactericidal agent against various pathogenic bacterial strains. This eco-friendly and nontoxic method is highly reproducible and has further benefits like compatibility with pharmaceutical and biomedical applications. Furthermore, these phytosynthesized AgNPs can be conjugated with various drugs, antibodies, or any ligand of interest for more powerful imaging, drug delivery, and diagnostic techniques for the service of mankind. But, before using them, a deliberate calculation of sub-lethal concentrations of prepared AgNPs 
is recommended for their use in any medical applications, especially those that involve pathogenic bacteria.

\section{AUTHOR CONTRIBUTIONS}

All authors made substantial contributions to conception and design, acquisition of data, or analysis and interpretation of data; took part in drafting the article or revising it critically for important intellectual content; agreed to submit to the current journal; gave final approval of the version to be published; and agree to be accountable for all aspects of the work. All the authors are eligible to be an author as per the international committee of medical journal editors (ICMJE) requirements/guidelines.

\section{FUNDING}

There is no funding to report.

\section{CONFLICTS OF INTEREST}

The authors report no financial or any other conflicts of interest in this work.

\section{ETHICAL APPROVALS}

This study does not involve experiments on animals or human subjects.

\section{PUBLISHER'S NOTE}

This journal remains neutral with regard to jurisdictional claims in published institutional affiliation.

\section{REFERENCES}

1. Raj DR, Prasanth S, Vineeshkumar TV, Sundarsanakumar C. Surface plasmon resonance based fiber optic dopamine sensor using green synthesized silver nanoparticles. Sen Act B Chem 2016;224:600-6.

2. Mathur P, Jha S, Ramteke S, Jain NK. Pharmaceutical aspects of silver nanoparticles. Artif Cells Nanomed Biotechnol 2017;12:1-12.

3. Baghayeri M, Mahdavi B, Abadi ZHM, Fardahi S. Green synthesis of silver nanoparticles using water extract of Salvia leriifolia: antibacterial studies and applications as catalysts in the electrochemical detection of nitrite. Appl Organometal Chem 2018;32:1-9.

4. Ge L, Li Q, Wang M, Ouyang J, Li X, Xing MM. Nanosilver particles in medical applications: synthesis, performance, and toxicity. Int J Nanomed 2014;9(1):2399-407.

5. Moldovan B, David L, Vulcu A, Olenic L, Perde-Schrepler M, FischerFodor $\mathrm{E}$, et al. In vitro and in vivo anti-inflammatory properties of green synthesized silver nanoparticles using Viburnum opulus L. fruits extract. Mater Sci Eng C 2017;79:720-7.

6. Li J, Wang Y, Liang R, An X, Wang K, Shen G, et al. Recent advances in targeted nanoparticles drug delivery to melanoma. Nanomedicine 2015;11(3):769-94.

7. Yeasmin S, Datta HK, Chaudhuri S, Malik D, Bandyopadhyay A. In-vitro anti-cancer activity of shape controlled silver nanoparticles (AgNPs) in various organ specific cell lines. J Mol Liq 2017;242:757-66.

8. Agnihotri S, Mukherji S, Mukherji S. Size-controlled silver nanoparticles synthesized over the range 5-100 $\mathrm{nm}$ using the same protocol and their antibacterial efficacy. RSC Adv 2014;4:3974-83.

9. Handoko CT, Huda A, Gulo F. Synthesis pathway and powerful antimicrobial properties of silver nanoparticle: a critical review. Asian J Sci Res 2019;12:1-17.
10. Govindappa M, Hemashkhar B, Arthikala MK, Rai VR, Ramachandra YL. Characterization, antibacterial, antioxidant, antidiabetic, antiinflammatory and antityrosinase activity of green synthesized silver nanoparticles using Calophyllum tomentosum leaves extract. Results Phys 2018;9:400-8.

11. Siddiqi KS, Husen A, Rao RAK. A review on biosynthesis of silver nanoparticles and their biocidal properties. J Nanobiotechnol 2018;16:14:1-28.

12. Hamouda RA, Hussein MH, Abo-Elmagd RA, Bawazir SS. Synthesis and biological characterization of silver nanoparticles derived from the cyanobacterium Oscillatoria limnetica. Sci Rep 2019;9(1):13071; doi:10.1038/s41598-019-49444-y

13. Singhal G, Bhavesh R, Kasariya K, Sharma AR, Singh RP. Biosynthesis of silver nanoparticles using Ocimum sanctum (Tulsi) leaf extract and screening its antimicrobial activity. J Nanopart Res 2011;13:1-8.

14. Ahmed S, Ahmad M, Swami BL, Ikram S. A review on plants extract mediated synthesis of silver nanoparticles for antimicrobial applications: a green expertise. J Adv Res 2016;7:17-28.

15. Gopinatha V, Ali MD, Priyadarshini S, Meera PN, Thajuddinb N, Velusamy P. Biosynthesis of silver nanoparticles from Tribulus terrestris and its antimicrobial activity: a novel biological approach. Colloid Surf B: Biointerface 2012;96:69-74.

16. Hui Y, Yan-yu R, Tao W, Chuang W. Preparation and antibacterial activities of $\mathrm{Ag} / \mathrm{Ag}+/ \mathrm{Ag} 3 \pm$ nanoparticle composites made by pomegranate (Punica granatum) rind extract. Results Phys 2016;6:299-304.

17. Vincent MS, Uphoff S. Bacterial phenotypic heterogeneity in DNA repair and mutagenesis. Biochem Soc Trans 2020;48:451-62.

18. Salem W, Leitner DR, Zingl FG, Schratter G, Prassl R, Goessler W, et al. Antibacterial activity of silver and zinc nanoparticles against Vibrio cholerae and enterotoxic Escherichia coli. Int J Med Microbiol 2015;305:85-95.

19. Sanchez-Lopez E, Gomes D, Esteruelas G, Bonilla L, Lopez-Machado $\mathrm{AL}$, Galindo R, et al. Metal-based nanoparticles as antimicrobial agents: an overview. Nanomaterials 2020;10292:1-39.

20. Isaac RSR, Nair AS, Varghese E, Chavali M. Phytochemical, antioxidant and nutrient analysis of medicinal rice (Oryza sativa L.) varieties found in South India. Adv Sci Lett 2012;5:1-5.

21. Suriyavathana M, Roopavathi I, Vijayan V. Phytochemical characterization of Triticum aestivum (Wheat grass). J Pharm Phytochem 2016;5(1):283-6.

22. Rajeev K, Jaydeep S. The "Green blood" wheat grass, a health tonic having antibacterial potential. J Pharm Res 2015;4:46-54.

23. Murali M, Raj MA, Akhil SA, Liji RS, kumar SS, Nair AM, et al. Preliminary phytochemical analysis of wheat grass leaf extracts. Int $\mathrm{J}$ Pharm Sci Rev Res 2016;40(1):307-12.

24. Lehrer RI, Rosenman M. Ultrasensitive assays for endogenous antimicrobial polypeptides. J Microbiol Methods 1991;137:167-73.

25. Pahal V, Kaur A, Dadhich KS. Effect of combination therapy using cow (Bos indicus) urine distillate and some Indian medicinal plants against selective pathogenic Gram-negative bacteria. Int J Pharm Sci Res 2017;8(5):2134-42.

26. Al-Bakri GA, Afifi FU. Evaluation of antimicrobial activity of selected plant extracts by rapid XTT colorimetry and bacterial enumeration. J Microbiol Methods 2007;68:19-25.

27. Shikha S, Chaudhuri SR, Bhattacharyya MS. Facile one pot greener synthesis of sophorolipid capped gold nanoparticles and its antimicrobial activity having special efficacy against Gram negative Vibrio cholerae. Sci Rep 2020;10:1463; doi:10.1038/s41598-01957399-3

28. Dong Y, Zhu H, Shen Y, Zhang W, Zhang L. Antibacterial activity of silver nanoparticles of different particle size against Vibrio natriegens. PLoS One 2019;14(9):e0222322; doi:10.1371/journal.pone.0222322

29. Qais QFA, Shafiq A, Khan HM, Husain FM, Khan RA, Alenazi B, et al. Antibacterial effect of silver nanoparticles synthesized using 
Murraya koenigii (L.) against multidrug-resistant pathogens. Bioinorg Chem Appl 2019;4649506:1-12; doi:10.1155/2019/4649506

30. Das K, Tiwari RKS, Shrivastava DK. Techniques for evaluation of medicinal plant products as antimicrobial agent: current methods and future trends. J Med Plants Res 2010;4:104-11.

31. Kumar R, Ghoshal G, Jain A, Goyal M. Rapid green synthesis of silver nanoparticles (AgNPs) using (Prunus persica) plants extract: exploring its antimicrobial and catalytic activities. J Nanomed Nanotechnol 2017;8(8):2157-7439.

32. Arumai SD, Mahendiran D, Senthil KR, Kalilur RA. Garlic, green tea and turmeric extracts-mediated green synthesis of silver nanoparticles: phytochemical, antioxidant and in vitro cytotoxicity studies. J Photochem Photobiol B 2018;180:243-52.

33. Anandalakshmi K, Venugobal J, Ramasamy V. Characterization of silver nanoparticles by green synthesis method using Pedalium murex leaf extract and their antibacterial activity. Appl Nanosci 2015;6(3):399-408; doi:10.1007/s13204-015-0449-z.

34. Amerkhanova SK, Shlyapov RM, Afanas'ev DA, Uali AS. The optical and sorption properties of films of polyvinyl alcohol with silver nanoparticles. Plasticheskie Massy 2012;3:12-4.

35. Pirtarighat S, Ghannadnia M, Baghshahi S. Green synthesis of silver nanoparticles using the plant extract of Salvia spinosa grown in vitro and their antibacterial activity assessment. J Nanostructure Chem 2019;9:1-9.

36. Zuas O, Hamim N, Sampora Y. Bio-synthesis of silver nanoparticles using water extract of Myrmecodia pendans (Sarang Semut plant). Mater Lett 2014;123:156-9.

37. Mariselvam R, Ranjitsingh AJA, Nanthini UR, Kalirajan K, Padmalatha C, Selvakumar MP. Green synthesis of silver nanoparticles from the extract of the inflorescence of Cocos nucifera (Family: Arecaceae) for enhanced antibacterial activity. Spectrochim Acta Part A: Mol Biomol Spectrosc 2014;129:537-41.

38. Suarez-Cerda J, Alonso-Nunez G, Espinoza-Gomez H, FloresLopez LZ. Synthesis, kinetics and photocatalytic study of "ultrasmall" Ag-NPs obtained by a green chemistry method using an extract of Rosa 'Andeli' double delight petals. J Colloid Interface Sci 2015;458:169-77.

39. Ramyal M, Subapriya MS. Green synthesis of silver nanoparticles. Int J Pharm Med Biol Sci 2012;1(1):54-61.

40. Xiu ZM, Zhang QB, Puppala HL, Colvin, VL, Alvarez PJ. Negligible particle-specific antibacterial activity of silver nanoparticles. Nano Lett 2012;12:4271-5.

41. Chwalibog A, Sawosz E, Hotowy A, Szeliga J, Mitura S, Mitura K, et $a l$. Visualization of interaction between inorganic nanoparticles and bacteria or fungi. Int J Nanomed 2010;5:1085-94.

42. Prabhu S, Poulose EK. Silver nanoparticles: mechanism of antimicrobial action, synthesis, medical applications, and toxicity effects. Int Nano Lett 2012;2:32-42.

43. Zhang Y, Yang D, Kong Y, Wang X, Pandoli O, Gao G. Synergetic antibacterial effects of silver nanoparticles @ Aloe vera prepared via a green method. Nano Biomed Eng 2010;2(4):252-7.

44. Loo YY, Rukayadi Y, Nor-Khaizura MAR, Kuan CH, Chieng $\mathrm{BW}$, Nishibuchi $\mathrm{M}$, et al. In vitro antimicrobial activity of green synthesized silver nanoparticles against selected Gram-negative foodborne pathogens. Front Microbiol 2018;9:1555; doi:10.3389/ fmicb.2018.01555

45. Jha D, Thiruveedula PK, Pathak R, Kumar B, Gautam HK, Agnihotri $\mathrm{S}$, et al. Multifunctional biosynthesized silver nanoparticles exhibiting excellent antimicrobial potential against multi-drug resistant microbes along with remarkable anticancerous properties. Mater Sci Eng C 2017;80:659-69.

46. Bader A, Alsalme A, Ahmad I. Antibacterial effect of silver nanoparticles synthesized using Murraya koenigii (L.) against multidrug-resistant pathogens. Bioinorg Chem Appl 2019;4649506:112; doi:10.1155/2019/4649506
47. Liao SL, Zhang Y, Pan X, Zhu F, Jiang C, Liu Q, et al. Antibacterial activity and mechanism of silver nanoparticles against multidrugresistant Pseudomonas aeruginosa. Int J Nanomed 2019;14:1469-87.

48. Pal S, Tak YK, Song JM. Does the antibacterial activity of silver nanoparticles depend on the shape of the nanoparticle? A study of the Gram-negative bacterium Escherichia coli. Appl Environ Microbiol 2007;73:1712-20.

49. Tang S, Zheng J. Antibacterial activity of silver nanoparticles: structural effects. Adv Healthcare Mater 2018;1701503:1-10.

50. Hashimoto MC, Prates RA, Kato IT, Nunez SC, Courrol LC, Ribeiro MS. Antimicrobial photodynamic therapy on drug-resistant Pseudomonas aeruginosa-induced infection: an in vivo study. Photochem Photobiol 2012;88:590-5.

51. Tanase C, Berta L, Coman NA, Roșca I, Man A, Toma F, et al. Antibacterial and antioxidant potential of silver nanoparticles biosynthesized using the spruce bark extract. Nanomaterials (Basel) 2019;9(11):1541.

52. Guzman M, Dille J, Godet S. Synthesis and antibacterial activity of silver nanoparticles against Gram-positive and Gram-negative bacteria. Nanomedicine 2012;8:37-45.

53. Auffan M, Rose J, Bottero JY, Lowry GV, Jolivet JP, Wiesner MR. Towards a definition of inorganic nanoparticles from an environmental, health and safety perspective Nat Nanotechnol 2009;4(10):634-41.

54. Lara HH, Ayala-Nunez NV, Turrent LDCI, Padilla CR. Bactericidal effect of silver nanoparticles against multidrug-resistant bacteria. World J Microbiol Biotechnol 2010;26:615-21.

55. Taner M, Sayar N, Yulug IG, Suzer S. Synthesis, characterization and antibacterial investigation of silver-copper nanoalloys. J Mater Chem 2011;21:13150-4.

56. Krychowiak M, Grinholc M, Banasiuk R, Krauze-Baranowska M, Glod D, Kawiak A, et al. Combination of silver nanoparticles and Drosera binata extract as a possible alternative for antibiotic treatment of burn wound infections caused by resistant Staphylococcus aureus. PLoS One 2014;9: e115727.

57. Schacht VJ, Neumann LV, Sandhi SK, Chen L, Henning T, Klar PJ, et al. Effects of silver nanoparticles on microbial growth dynamics. J Appl Microbiol 2012;114:25-35.

58. Guo Z, Chen G, Zeng G, Huang Z, Chen A, Hu L, et al. Cysteineinduced hormesis effect of silver nanoparticles. Toxicol Res 2016;5:1268-72.

59. Iavicoli I, Leso V, Fontana L, Calabrese EJ. Nanoparticle exposure and hormetic dose-responses: an update. Int J Mol Sci 2018;19(805):1-23.

60. Rodrigues DF, Elimelech M. Toxic effects of single-walled carbon nanotubes in the development of E. coli biofilm. Environ Sci Technol 2010;44:4583-9.

61. Calabrese EJ, Baldwin LA. Defining hormesis. Hum Exp Toxicol 2002;21(2):91-7.

62. Zimmermann A, Bauer MA, Kroemer G, Madeo F, Carmona-Gutierrez $\mathrm{D}$. When less is more: hormesis against stress and disease. Microbial Cell 2014;1(5):150-3.

63. Torres-Barcelo C, Kojadinovic M, Moxon R, MacLean RC. The SOS response increases bacterial fitness, but not evolvability, under a sublethal dose of antibiotic. Proc R Soc B 2015;282:20150885.

64. Bakkeren E, Diard M, Hardt WD. Evolutionary causes and consequences of bacterial antibiotic persistence. Nat Rev Microbiol 2020;18(9):479-90; doi:10.1038/s41579-020-0378- z.

65. Baharoglu Z, Mazel D. SOS, the formidable strategy of bacteria against aggressions. FEMS Microbiol Rev 2014;38:1126-45.

66. Barrett TC, Mok WWK, Murawski AM, Brynildsen MP. Enhanced antibiotic resistance development from fluoroquinolone persisters after a single exposure to antibiotic. Nat Commun 2019;10:1177; doi:10.1038/s41467-019-09058-4

67. Goormaghtigh F, Melderen LV. Single-cell imaging and characterization of Escherichia coli persister cells to ofloxacin in 
exponential cultures. Sci Adv 2019;5:eaav9462; doi:10.1126/sciadv aav9462.

68. Fridman O, Goldberg A, Ronin I, Shoresh N, Balaban NQ. Optimization of lag time underlies antibiotic tolerance in evolved bacterial populations. Nature 2014;513:418-21.

69. Shi J, Huber M, Wang T, Dali W, Lin Z, Chun-Sheng Y. Progress in the studies on hormesis of low-dose pollutants. Environ Dis 2016;1:58-64.

70. Levin-Reisman I, Ronin I, Gefen O, Braniss I, Shoresh N, Balaban NQ. Antibiotic tolerance facilitates the evolution of resistance. Science 2017;355:826-30.

71. Stokes JM, Lopatkin AJ, Lobritz MA, Collins JJ. Bacterial metabolism and antibiotic efficacy. Cell Metab 2019;30:251-9.
How to cite this article:

Pahal V, Kumar P, Kumar P, Kumar V. Antibacterial activity and the hormetic response of silver nanoparticles synthesized using leaflet extract of wheat (Triticum aestivum) and rice (Oryza sativa) crop plants. J Appl Biol Biotech 2022; 10(02):154-167. 